\title{
Process intensification connects scales and disciplines towards sustainability
}

\author{
Daria C. Boffito, ${ }^{\text {a David Fernandez-Rivas }}{ }^{\mathrm{b}}$ \\ ${ }^{a}$ Canada Research Chair in Intensified Mechano-Chemical Processes for Sustainable Biomass \\ Conversion, Polytechnique Montréal, Chemical Engineering Department, C.P. 6079, succ. \\ Centre-ville, Montréal, QC, Canada H3C 3A7 \\ ${ }^{b}$ Mesoscale Chemical Systems Group, MESA+ Institute and Faculty of Science and Technology, \\ University of Twente, Enschede 7522NB, The Netherlands
}

\section{Correspondence}

Daria C. Boffito

Canada Research Chair in Intensified Mechano-Chemical Processes for Sustainable Biomass Conversion, Polytechnique Montréal, Chemical Engineering Department, C.P. 6079, succ. Centre-ville, Montréal, QC, Canada H3C 3A7

Email: daria-camilla.boffito@polymtl.ca

\begin{abstract}
Process intensification (PI) has been established as a cluster of technologies able to produce more with less. While scientists around the globe advocate for new semantics that are increasingly tied to the notion of sustainability, what does the literature data say about PI? A Vosviewer bibliometric map of PI displays it as closely linked to the subjects of design, optimization, gas-to-liquid technologies, mass transfer, catalysis, and kinetics. We analyze the relationship between PI and these subjects while identifying misconceptions about the intensifying potential of some of them, as is the case for optimization. We provide examples and
\end{abstract}

This article has been accepted for publication and undergone full peer review but has not been through the copyediting, typesetting, pagination and proofreading process which may lead to differences between this version and the Version of Record. Please cite this article as doi: $10.1002 /$ cjce. 23871 
summarize the recent technological trends for all these cases. Finally, we provide an outlook on the future of PI in which we identify elements that will be key to accelerate the adoption of PI at the commercial scale.

\section{INTRODUCTION}

What we understand as process intensification (PI) today has existed since well before it was given a name. It first embraced fields of application related to chemical engineering but also aerospace and photography. The first publications on PI were patents that date back to the 1930s and concerned HiGee forces, ie, equipment design combined with high speed rotation. The main result was high gravity momentum to overcome limitations imposed by gravity and improve separation and micromixing. In 1935, Podbielniak ${ }^{[1]}$ realized that the curse of gravity causes distillation columns to be tall, which led him to conceive of a spinning device (HiGee) with a tube coiled around a conical support mounted on a turntable. After this first invention, Podbelniak's patenting activity on HiGee spanned 35 years with a coherent aim of shrinking distillation column sizes by 10, 100, or 1000 times. The National Aeronautics and Space Administration (NASA) was clearly a significant player in the development of HiGee technologies, in particular the rotating heat pipes, since zero-gravity limits heat and mass transfer, while HiGee devices generate centrifugal accelerations that are useful in a number of cases in a spacecraft. ${ }^{[2]}$ In 1935 , a patent by Hickman, ${ }^{[3]}$ with Kodak as assignee, described how to produce high-grade plastic films on a spinning disk mounted on a rotary still. The aim was to produce safer photographic rolls several times faster than the traditional cellulose acetate-based safety films and the transparent plastic rolls made from highly flammable nitrocellulose, also called nitrate film. It was however Ramshaw and his team ${ }^{[4,5]}$ at Imperial Chemical Industries (ICI) in the 1970s-1980s that designed HiGee technologies that reached their full potential by radically reducing the size of chemical equipment. ICI designs included the HiGee rotating gas/liquid contactor packed bed, the printed circuit heat exchanger, and the Rotex absorption heat pump, among others. ${ }^{[4,5]}$ 
It was in 1995, during the first International Conference on Process Intensification for the Chemical Industry, that Ramshaw ${ }^{[6]}$ defined PI as a strategy to dramatically reduce $(>100 \mathrm{x})$ the size of a chemical plant, while maintaining a target production objective. In 2000, Stankiewicz and Moulijn ${ }^{[7]}$ proposed a wider definition, describing PI as "any chemical engineering development that leads to a substantially smaller, cleaner, and more energy efficient technology.” Other authors proposed different but similar definitions, mentioning “smaller” and “more efficient technologies,"[8] "radically innovative principles to reduce costs, energy requirements and waste,”[9] and “an integrated approach.”[10] Van Gerven and Stankiewicz ${ }^{[11]}$ introduced a classification by four different approaches that either individually or combined can achieve various degrees of PI. These approaches implement PI by taking action in different domains, namely (a) structure (spatial domain), (b) energy (thermodynamic domain), (c) synergy (functional domain), and (d) time (temporal domain). ${ }^{[11]}$ Lastly, updated definitions of PI emerged during the workshop titled “Education on Process Intensification” on 3-7 June 2019. There, a group of experts with multidisciplinary backgrounds encompassing academia, industry, governmental agencies, technical societies, and institutes met at the Lorentz Center (Leiden, NL) to set guidelines on how to implement PI education in academia, industry, and other settings. Two papers were written by participants on the outcomes of the workshops. ${ }^{[12,13]}$ The two definitions given by Fernandez Rivas et $\mathrm{a}^{[12,13]}$ are different from definitions provided previously that are related to size and efficiency. These two definitions set the focus on new elements:

1. Process intensification is an approach by function a departure from the conventional process design by unit operations. This approach focuses not only on the process itself but also on what happens outside or as a consequence of the process. With the approach by function, unit operations such as reactor, heat exchanger, and distillation column, for instance, become reaction, heating, and separation. This shifts the focus from the unit to a function that can be combined with others and achieved in the same process element. Enhancing heat and mass transfer by either burning more fuel or increasing heating fluids flow or shear rates is a way of 
increasing productivity by traditional unit operations. However, this approach affects the environment through increased emissions, on the process economics through increased fixed and operating costs, and on the complexity of the processes through increased technical and safety risks. On the other hand, PI offers strategies to design more efficient systems by acting on process requirements (heat and mass transfer) without negatively affecting the external variables, as these are related to environment, investment, and process cost.

2. As such, PI is established as a means of achieving sustainability in the chemical industry and can potentially contribute to the United Nations Sustainable Development Goals. Indeed, the "I" in "PI" could at times be interpreted as innovation or improvement when the process employs one of the four approaches mentioned earlier. ${ }^{[12,13]}$

In the Canadian landscape, the Chemistry Industry Association of Canada (CIAC) leads and provides guidance to member companies on key issues including innovation, safety, and environment. One of the main focuses of the CIAC is the UN-recognized sustainability Responsible Care initiative, founded in Canada in 1985 and that is now implemented in 73 countries. CIAC's advocacy employs circular economy (CE) and life cycle analysis (LCA), ${ }^{[14]}$ which responds to the second item of the updated PI definition given above, ie, an approach that focuses not only on the process itself but also on what happens outside or as a consequence of it. In the literature there are several examples of reviews dedicated to PI that are technology specific. Examples include static mixers, ${ }^{[15-17]}$ cavitational reactors, ${ }^{[18]}$ flow reactors, ${ }^{[19-24]}$ membrane gas separation and reactors, ${ }^{[25-27]}$ intensified separations, ${ }^{[28,29]}$ microengineered reactors, ${ }^{[30-37]}$ microwave-assisted chemistry, ${ }^{[22,38,39]}$ low frequency ultrasound processes, ${ }^{[40-42]}$ to cite a few. There are, however, fewer reviews on PI as an innovative approach to chemical engineering design in general, each focusing on different aspects of it. These include PI principles, ${ }^{[11]}$ classification of PI equipment and methods, ${ }^{[7]}$ overview of PI methods, ${ }^{[43]}$ the relationship between PI and process system engineering (PSE) and process control, ${ }^{[44]}$ systematic approaches to PI, ${ }^{[45]}$ the relationship between PI and materials, ${ }^{[46]}$ and alternative energy sources as a PI approach. ${ }^{[47]}$ This review concerns PI as an innovative concept of chemical engineering, 
and rather than focusing on specific PI equipment or methods, it attempts to identify the technological trends in the literature and defines the multidisciplinary links among those. We believe that these links are key in identifying the potential for PI to contribute to sustainability.

\section{BIBLIOMETRICS OF PROCESS INTENSIFICATION}

The Web of Science Core Collection (WoS) indexed over 3480 documents between 1990-2019 that contained process intensification under the basic search category topic. Of these 3480+ documents, 877 belonged to the 2018-2019 period only. Since 1992, the number of papers on process intensification has increased exponentially with $N_{\text {art }}=10^{\wedge}(0.0791 \mathrm{y}-157)$, where $N_{\text {art }}$ is the total number of papers and y is the publication year (Figure 1).

PI also increasingly demonstrates its potential to crosscut multidisciplinary chemical engineering. VOSviewer12 created a word map of the top 100 keywords in articles on the topic of process intensification from the WoS search mentioned earlier (Figure 2). The sizes of the circles correlate with frequency of the keywords in the articles and the color regroups similar subjects, while lines of different thicknesses also connect subjects that are related. The largest circle, ie, the most frequent keyword appearing in PI articles, was mass transfer (389), followed by design (296), reactor (269), optimization (251), and mass transfer (110). The smallest circles correspond to 35 occurrences (eg, two-phase flow, residence time distribution, etc.). Among the WoS categories, chemical engineering was the top category with 2232 occurrences, followed by energy and fuels (670), multidisciplinary chemistry (444), environmental engineering (355), biotechnology and applied microbiology (250), physical chemistry (240), green and sustainable science and technology (176).

In the following sections we take a closer look at the five subjects (macro-groups) that the bibliometric map in Figure 2 displays in different colors, and we detail the relationship between PI and each of this subjects with the most up-to-date examples from the literature. 


\section{PROCESS INTENSIFICATION AND MASS TRANSFER}

Mass transfer is the most recurring keyword related to process intensification (PI). This is not surprising as transport phenomena are at the basis of all chemical processes. The primary aim of PI is to curtail all transfer limitations to reduce the rate of the processes to their intrinsic reactions rates. ${ }^{[48]}$ Therefore, most process-intensifying equipment and methods aim to maximize the mass transfer at different levels, from the molecular to the macroscopic level (from picoseconds to hour time scales and from Armstrong to meter scales; see Section 4). This is also evident from the four principles (goals) of PI first identified by Van Gerven and Stankiewicz ${ }^{[11]}$ : (a) to maximize both the molecular and macroscale transport phenomena of heat, momentum, and mass, (b) to maximize the specific surface area to which these forces apply in order (c) to give each molecule the same processing experience, and (d) to maximize synergistic effects by combining unit operations (reaction, distillation, mixing). ${ }^{[11]}$ In principles a-c, transport phenomena are the target elements that PI focuses on, making it evident that PI benefits most of those processes that are limited by transport rates. Chen ${ }^{[49]}$ reported a non-exhaustive list of some process-intensifying by mass transfer and heat transfer capacity equipment (Figure 3). Most process-intensifying equipment and methods offer a way of minimizing mass and heat transfer limitations. ${ }^{[7]}$ Providing the details of these is beyond the scope of this paper, therefore we will only cite a few examples with reference to the bibliometric map in Figure 2.

\subsection{Static mixers}

Static mixers are structured filling bodies that can be incorporated in both static and rotating devices (eg, in absorption columns or rotating packed beds, RPBs) with the aim of increasing the interfacial area of heat and mass transfer. The most popular filling bodies are Katapak-SP and Sulzer-BX. Stec and Synowiec ${ }^{[50]}$ characterized the pressure drop, the residence time distribution, ${ }^{[51]}$ and the mixture homogeneity of two types of static mixers, Koflo and Kenics. ${ }^{[52]}$ Static mixers as a PI technology do not offer direct thermodynamic advantages over empty columns; however, concentration and temperature are homogeneous over the column cross 
section, thus providing the same thermodynamic conditions throughout the unit. Static mixers are an ubiquitous example of a PI technology that industry has adopted commercially with significant success. ${ }^{[12]}$

\subsection{Rotating packed bed (RPB) reactors (HiGee reactors)}

Wang et $\mathrm{al}^{[53]}$ recently reviewed the latest developments on mass transfer in rotating packed bed (RPB) reactors. They conclude that two-stage counter-current RPB reactors (TSCC-RPB) are the type of RPBs preferred for processes limited by reaction rate. Wenzel and Górak ${ }^{[54]}$ reviewed micromixing in RPBs and concluded that RBP reactors ensure rapid micromixing even in the presence of high liquid flow rates and high viscosities. Also, when there is more than one liquid to feed to the RPB, the feeding rate to the reactor should be as similar as possible to avoid segregation. On the other hand, when segregation is not a problem, counter-current flows equalize concentrations in the unit to reduce the associated entropy gain. ${ }^{[4]}$ Boffito and Van Gerven $^{[48]}$ observed that most examples in the literature in the context of PI and catalysis on RPBs deal with CFD simulations rather than the actual experiments. ${ }^{[48]}$ Most reviews on RPBs agree that current CFD simulations, even when aided by visual tools, are still limited in characterizing the behavior of RPB, and that energy density and efficiency, as well as the efficiency of mixing, are parameters that often lack quantification. Examples in the literature where the rate improved when adopting a RPB include natural gas dehydration, ${ }^{[55]} \mathrm{CO}_{2}$ capture with amine absorbers, ${ }^{[56]} \mathrm{H}_{2} \mathrm{~S}$ removal by oxidative absorption, ${ }^{[57]}$ enzymatic transesterification, ${ }^{[58]}$ and sulfonation of aromatics. ${ }^{[59]}$

\subsection{Spinning disc reactors}

In a spinning disc reactor (SDR), a liquid flows vertically through a channel perpendicular and central to a rotating surface. The liquid then flows towards the perimeter of the rotating disk, thus creating a micrometer-wide thin film. From a thermodynamic point of view, SDRs provide high heat transfer rates across the disc, which depends on the disk material, the channel width, and the heat-transfer fluid. ${ }^{[60]}$ Spinning discs reactors represent one of the first innovations in PI (HiGee 
equipment). However, most applications are in the field of mechanical engineering with the aim of efficiently transfer fluids, rather than accelerating reactions. For applications in chemical engineering and chemicals synthesis, the disk usually carries a catalyst; the thin film and the catalyst are then in intimate contact. SDRs applications to reactions include production of polymers, ${ }^{[61,62]}$ synthesis of pharmaceutical products (eg, Darzen and Knoevenagel’s reaction), ${ }^{[60]}$ and photocatalytic applications. ${ }^{[63]}$

\subsection{Oscillatory baffled reactors (OBRs)}

In addition, OBRs, also called pulsed (vertical or horizontal), are columns containing plates (baffles). The baffle is often a single central opening (ie, an integral baffle), but there are other configurations such as helical baffles. ${ }^{[64]}$ One of the characteristics of OBRs is that while the fluid flow stays laminar, it is the radial and axial components generated by pushing the fluid through the annular region whose velocity is of the same magnitude of the forward flow that generate the turbulent vortices (eddies) that are typical of OBRs and generate eddy mixing. When the fluid oscillates at frequencies between $0.5 \mathrm{~Hz}-6 \mathrm{~Hz}$ and amplitudes between $1 \mathrm{~mm}-100$ $\mathrm{mm}$, this generates a periodical reverse fluid wave that interacts with the eddies created by the passage of the liquid in the forward motion through the baffles, thus achieving micromixing. The key is selecting the oscillation frequency and amplitude of the laminar flow to control the degree of mixing with high precision. ${ }^{[48,65]}$ McDonough et al ${ }^{[64]}$ reviewed the latest developments in OBRs. In particular, the authors focused on the mesoscale OBR (meso-OBR, $\mathrm{d}>15 \mathrm{~mm}$ ), which functioned as an ideal plug flow reactor at flow rates between $0.3 \mathrm{~mL} / \mathrm{min}-8 \mathrm{~mL} / \mathrm{min}$, ie, low to moderate. ${ }^{[64]}$ The same authors also compared the quality of micromixing for baffles of different geometries. ${ }^{[66]}$ Thermodynamically speaking, heat transfer in OBRs is time-dependent. For instance, when plotted against the residence time, the Nusselt number $(\mathrm{Nu})$ and the bulk temperature give time-periodic solutions. For different geometries the heat transfer depends most on the flow amplitude, whereby the maximum heat transfer occurs at amplitudes of the same lengths as the opening of the baffles. ${ }^{[67]}$ Examples of applications of OBRs include esterification and transesterification to produce biodiesel ${ }^{[68-70]}$ and biolubricants, ${ }^{[71]}$ imine synthesis to prove 
the suitability of OBRs to rapidly obtain kinetics, ${ }^{[72]}$ butylation, ${ }^{[73]}$ photooxidation, ${ }^{[74]}$ crystallization, ${ }^{[75,76]}$ and testing gas-liquid mass transfer. ${ }^{[77]}$ Fernandez-Rivas et al ${ }^{[78]}$ calculated a total intensification factor, $\mathrm{IF}_{\text {total }}=19.44$, for an $\mathrm{OBR}$ with a volume and residence time three and two orders of magnitude lower than a batch reactor, respectively. We will discuss the intensification factor further in Section 8.

\subsection{Microreactors}

Microreactors are process-intensifying equipment that promote transport phenomena (Figure 7). We will discuss this technology in detail in Section 6.

\section{PROCESS INTENSIFICATION AND DESIGN}

The second most recurrent topic linked to process intensification (PI) is “design”, followed by “optimization”, and then "system”. Intensifying through equipment design, such as reactor design (eg, microreactors), heat exchangers (eg, compact heat exchangers), and distillation columns (eg, dividing wall columns) is a prevailing approach to increase transport phenomena rates. However, process optimization and process intensification are not the same. Process optimization is aimed at direct plant profitability in an immediately quantifiable way and targets existing process variables. ${ }^{[79]}$ For instance, real-time optimization (RTO) encompasses a sequence of steps, including modelling, numerical optimization, and application of models as an input to the process to modify it while complying with its constraints. ${ }^{[80]} \mathrm{PI}$, in contrast, refers to innovative processes and methods that are well beyond the reach of traditional engineering optimization and incremental research and development. ${ }^{[12]}$ However, optimization, in particular software optimization, can help to accelerate the widespread application of PI at the commercial scale, in particular concerning reactor geometry. ${ }^{[81]}$

Concerning the third most frequent keyword in the literature in this macro-area, i.e. "system,” we can see it as process system engineering (PSE). The latter is more pertinet to PI than the keyword 
“optimization”. Marquardt et al ${ }^{[82]}$ proposed a synergistic relationship between PI and PSE in which the two disciplines still remain independent. The same concept has since been reconsidered by other authors and expanded, for instance by Moulijn et al ${ }^{[83]}$ (Figure 4). While PI typically targets scale lengths of up to $1 \mathrm{~m}$ and process events of a maximum of 1 hour (which refers exclusively to intrinsic reaction rates, PSE, in its multidisciplinary nature), incorporates the whole value chain and covers lengths and time scales that characterize enterprises with a supply/consumer chain that covers kilometers in range. In general, enterprises encompass any business with multiple locations, divisions, or departments that collaborate to achieve business objectives. Therefore, PSE includes aspects related to chemical reaction engineering, control engineering, industrial engineering, and project management. PI groups all elements of PSE from molecules to process units in a single concept or approach by controlling reactive events at the molecular level, thus facilitating not only the scale-up of a given process but also the integration task of PSE to attain the enterprise's value chain.

We wish to highlight the close relationship between PSE and life cycle analysis (LCA), and therefore between PSE and PI. Bergerson et $\mathrm{a}^{[84]}$ recognize that emerging technologies, which is the case for PI technologies, are accompanied by certain issues when it comes to analyzing them through LCA. Indeed, the market for some of these technologies is also emerging. This uncertainty requires therefore a nuanced treatment, which provides a probabilistic distribution. For instance, Bergerson et al ${ }^{[84]}$ quantify the techno-economics versus the total $\mathrm{CO}_{2}$ emissions of some technologies to mitigate greenhouse gas emissions in North American refineries. These technologies include cogeneration of heat and power, carbon capture and sequestration, biomass gasification, and high temperature electrolysis to produce $\mathrm{H}_{2} \cdot{ }^{\left[{ }^{[8]}\right.}$ Published examples of the LCA approach from the same team include oil sand upgrading technologies by Pacheco et $\mathrm{a}^{[86]}$ and Sleep et $\mathrm{al}^{[87]}$ and greenhouse gas emissions and freshwater consumption from the Bakken tight oil by Laurenzia et al. ${ }^{[88]}$ 
To return to the initial relationship between PI and design, in the literature PI has been explored in design mainly for distillation and separation technologies (Figure 2). This should not come as a surprise. Despite distillation being the most practical fluid separation technology, it is both energy and capital intensive as the size and energy requirements increase with decreasing relative volatility. As a consequence, increasing the efficiency of distillation technologies has been a constant industrial endeavor in the past years. ${ }^{[89,90]}$ Reactive distillation (RD) and dividing-wall columns (DWCs) are the most common types of intensified separations (Figure 1). There are at least $150 \mathrm{RD}$ and 100 DWC commercially implemented distillation columns. ${ }^{\text {[91] }}$ India has been at the forefront of the application of DWC technologies, ${ }^{[92,93]}$ whereby the BPCL refinery of Mumbai was the world's first commercial example of a top DWC.

\subsection{Reactive distillation (RD)}

The most popular and perhaps simplest example of PI using equipment is reactive distillation (RD), ie, the combination of a reaction and distillation in a single vessel. ${ }^{[94]}$ The main processintensifying advantages of RD do not just derive from combining two functions in the same unit but also derive from the fact that the products are continuously removed, thus increasing the overall conversion in reactions thermodynamically limited by chemical equilibrium. The main challenges of $\mathrm{RD}$ concern the process control, as the process is non-linear. ${ }^{[95]}$ Hamsen $^{[96]}$ also reviewed the latest applications of $\mathrm{RD}$ in industry and quantified the economics in both reduced investment and energy requirements, which translated into $15 \%-80 \%$ cost savings compared to a traditional reactor-distillation column sequence. Catalytic distillation is a type of reactive distillation that often includes the adoption of a static mixing catalyst as a support, as described in the previous section.

\subsection{Dividing wall columns (DWC)}

Dividing wall columns (DWCs) (or Petlyuk-DWC) separate mixtures of several components. DWCs are distillation columns in which a vertical wall, whose position is determined by design, 
splits the central part of the column into two columns: the prefractionation column and the main column. The feed supplies the prefractionation column, while the medium boiling component separates from the mixture in the main column. The vapour coming from the stripping section splits into two and is directed to either the prefractionation column or the main column. Similarly, the liquid coming from the rectifying section splits into the two sections. The dividing wall can be placed at different locations in the column (Figure 5).

A dividing wall in the middle of the column may enrich a product stream without the need for additional reboiler energy. A top-DWC can usually replace an additional side-stripper downstream to the column, while a bottom-DWC replaces multiple columns in an indirect sequence. ${ }^{[93]}$ DWCs require less space and are 20\%-30\% less capital than conventional towers. ${ }^{[28,}$ ${ }^{93]}$ The efficiency of a single DWC is also close to that of a two-column sequence. ${ }^{\text {[93] }}$ From a thermodynamic point of view, a DWC does not present more advantages than a traditional Petlyuk column, unless there is heat transfer across the diving wall. An analysis of the column grand composite curve (CGCC) for both sections that the wall divides provides information on whether heat should be added or subtracted across the wall. ${ }^{[98]}$

\section{PROCESS INTENSIFICATION AND CATALYSIS}

Despite the fact that catalysis accelerates reaction rates and creates an alternate and more efficient reaction pathway/network, it is not, per se, a process intensification (PI) method as it does not involve the design of radically innovative technologies or the application of radically innovative methods. PI focuses on the overall process rate and productivity and acts on transport phenomena limitations, short-circuiting them, and thus reducing the overall process rate to its intrinsic reaction rates, uniquely governed by molecular events. However, in over $70 \%$ of the cases, in heterogeneous catalytic processes it is the intrinsic reaction rates that control the speed

of the entire process (limiting step). ${ }^{[99]}$ Therefore, PI and catalysis must be combined to achieve the highest process outputs possible. PI and catalysis can be combined in a number of ways. Boffito and Van Gerven ${ }^{[48]}$ recently reviewed examples of the integration of PI and catalysis and 
proposed a classification of their relationship based on the traditional approaches to PI by structure, energy, synergy, and time (Figure 6).

Heterogeneous catalysis can be integrated through both intensified equipment and intensified methods. While the integration of a catalyst in an equipment can be structured or unstructured, when a heterogeneous catalyst works in conjunction with a method (such as ultrasound or reactive distillation), it is often unstructured. Structured catalytic units are either organized in the reactor with a repeating geometry (such as multiple microchannel reactors operating in parallel and static mixers), or catalysts that are immobilized on a support (eg, on a spinning disk, see Figure 3). Unstructured catalysts include items such as catalytic powders and pellets. Synergy (in the functional domain) and time (in the temporal domain) are two approaches to PI that are always present when combining a catalyst with process intensifying equipment or method. ${ }^{[11]}$ In the functional domain, a catalyst is integrated with the process intensifying function of the selected equipment or method. In the temporal domain the aim is to minimize time scales of both extrinsic and intrinsic reaction rates by PI and catalyst design, respectively. Concerning the approach by structure, structuring catalytic units avoids spatial randomness, thus lessening preferential reaction pathways for transport phenomena that introduce inhomogeneities in the reactor geometry, such as hot spots and dead volumes. New technologies such as 3D printing make the design of specific catalyst geometries with controlled and repeatable micro and macro geometries possible. ${ }^{[100]}$ In general, for continuous processes, structured catalytic units are best suited as they can be designed in modular, replaceable components that can be substituted with new ones while they are being regenerated. Unstructured catalysts in combination with process-intensifying equipment or methods are easier to integrate when applying PI in the energy domain (Figure 2). This happens either because the equipment itself requires the catalyst to be in the form of powder or pellets (fluidized beds), or because often, at the laboratory scale, diagnostics or control of the geometry of irradiation (ultrasound or microwave) are poor. Therefore, integrating a catalyst in slurry increases the odds of the synergy between the 
intensified tool and the catalyst itself. However small, active catalyst particles often deactivate faster than larger pellets or catalytic units and are harder to recover and regenerate. ${ }^{[101]}$ Therefore, for continuous processes to scale-up, structured catalytic units may be the preferred choice.

\subsection{Process intensification and carbon capture}

$\mathrm{PI}$ and catalysis are closely linked in the design of reforming and $\mathrm{CO}_{2}$ capture technologies (Figure 1). This is because in recent years the research and development of gas-to-liquids (GtL) processes involving $\mathrm{CH}_{4}$ or $\mathrm{CO}_{2}$ capture and/or their transformation have been growing with the aim of providing solutions to decrease greenhouse gas (GHG) emissions. On the other hand, $\mathrm{H}_{2}$ and, in particular, clean technologies to produce $\mathrm{H}_{2}$ are key to both produce syngas from reverse water gas shift (RWGS) from $\mathrm{CO}_{2}$ and to enrich the same syngas to produce liquid fuels with the Fischer-Tropsch process. At the same time, smaller GtL enterprises (micro enterprises) have the potential to be established locally and provide services to a local/regional scale in a zerokilometer perspective. For instance, micro-GtL combine PI and numbering-up economics to reduce capital cost to convert wasted methane $\left(\mathrm{CH}_{4}\right)$ to syngas. ${ }^{[102,103]}$ Boffito, the first author of this paper, has contributed to designing a micro-GtL technology to convert flared and vented natural gas to value-added synthetic fuel. ${ }^{[104-107]}$ Indeed, converting waste associated natural gas is uneconomic with current GtL technologies. In our micro-GtL technology we target millisecond contact times in the catalytic partial oxidation of methane (CPOX) integrated with a tandem Fischer-Tropsch (FT) step to overcome the economic and remote process control constraints (Figure 7). ${ }^{[105]}$

Patience and Boffito ${ }^{[102]}$ recently reported a model that can be used to scale-up microtechnologies by numbering up and provided a micro-GtL case study. Traditional economic models either scale-up or scale-down the investment (C) for a given plant size (Q) with a power law, whereby the exponent $\alpha$ depends on the characteristic unit size (n). ${ }^{[102]}$ The model that 
Patience and Boffito proposed relies on the characteristic unit size (n), where the price of a modular plant relates to the cumulative capacity with an exponent $\beta=1 / \mathrm{n}-1$ called learning elasticity. For $10 \mathrm{bbl} / \mathrm{d}\left(1 \mathrm{bbl}=0.158987 \mathrm{~m}^{3}\right)$ modular microrefinery GtL units to convert wasted natural gas to Fischer-Tropsch fuels, the investment for 100 units approaches the cost of a single $1000 \mathrm{bbl} / \mathrm{d}$ unit, which is 1 million US dollars. ${ }^{[102]}$ Onel et al ${ }^{[108]}$ also proposed intensified $\mathrm{CH}_{4}$ steam methane reforming. They adopted a microchannel reactor and applied a multi-scale approach that included CFD modelling. They demonstrated that between $500 \mathrm{bbl} / \mathrm{d}-5000 \mathrm{bbl} / \mathrm{d}$ the intensified technology reduces the break-even oil prices by $\$ 10 / \mathrm{bbl}$.

Carbon capture and storage (CCS) technologies to capture $\mathrm{CO}_{2}$ can be categorized into three types: pre-combustion carbon capture, post-combustion carbon capture, and oxy-fuel carbon capture. They all possess limitations that PI has the potential to partially or fully overcome (Figure 8).

In pre-combust ion capture systems, $\mathrm{O}_{2}$ and/or steam gasifies a solid fuel (coal or biomass). Precombustion capture systems usually coincide with integrated gasification and combined cycle (IGCC) power plants. The gaseous products are then shift-converted to enrich them with syngas, which inevitably produces $\mathrm{CO}_{2}$. $\mathrm{CO}_{2}$ is then separated from $\mathrm{H}_{2}$ before it is combusted in a gas turbine to generate power. ${ }^{[109]}$ Since the $\mathrm{CO}_{2}$ concentration and pressure is higher in the output compared to post-combustion, the operating costs are lower, but the capital investment still remains high. ${ }^{[110]}$ To overcome high investment costs, PI offers some solutions. For instance Abdollahi et al ${ }^{[111]}$ proposed a catalytic membrane reactor integrated with the water gas-shift (WGS) unit to produce and separate $\mathrm{H}_{2}$. IGCC plants have an efficiency around $40 \%$ without the $\mathrm{CO}_{2}$ capture unit, while the efficiency drops to $32 \%$ for a $\mathrm{CO}_{2}$ capture of $90 \%$. Ahmed et al ${ }^{[112]}$ proposed an intensified pre-combustion capture system by removing the WGS step and the $\mathrm{CO}_{2}$ capture unit to burn the syngas directly, similarly to an oxy-fuel process. They obtained a reduction of $8.17 \%$ and $6.59 \%$ in the total investment and operations and maintenance (O\&M) 
costs, respectively, compared to the base case design. Recently, in the effort to make IGCC plants with a $\mathrm{CO}_{2}$ capture unit sustainable, Chen et $\mathrm{al}^{[113]}$ propose an integrated IGCC power plant with a membrane reactor and adsorptive reactor (MR-AR) combined. In this system, syngas flows through a membrane reactor (MR) filled with a WGS catalyst $\left(\mathrm{Co} / \mathrm{Mo} / \mathrm{Al}_{2} \mathrm{O}_{3}\right)$. The membrane separates $\mathrm{H}_{2}$ from the catalytic zone into multiple permeation tubes to yield high purity $\mathrm{H}_{2}$. The gases exiting the MR then flow in an AR filled with the same WGS catalyst and $\mathrm{H}_{2} \mathrm{~S}$-resitsant adsorbent hydrotalcite (Figure 9). The techno-economic analysis of this IGCC intensified plant calculated $9 \%+$ additional power and $16 \%+$ reduction in capital cost compared to traditional pre-combustion technology. ${ }^{[114]}$

The post-combustion carbon capture (PCC) has a high capital cost and the solvent regeneration step is energy intensive (the absorber and stripper account for $55 \%$ and $17 \%$ of the total capital cost, respectively). ${ }^{[115,116]}$ In this case, technologies such as rotating packed bed (RPB) absorbers, ${ }^{[115]}$ followed by stripping units, ${ }^{[117]}$ static mixers, and spinning disc reactors offer an opportunity to intensify the process. ${ }^{[115]}$ Recently, Zarei et al ${ }^{[118]}$ adopted a multi-layer perceptron artificial neural network (MLP-ANN) to quantify the effective gas-liquid interface in a rotating packed bed PCC by absorption. The available literature on these capture methods is, however, still limited. For instance, there is no literature data available on the integration of absorption and stripping, only reviews on the general subject of PCC. In conclusion, a combination of experimental tests, computational fluid dynamics (CFD) modelling (dynamic versus static) and particle image velocimetry (PIV) should be implemented to disclose the full potential of these process-intensifying technologies to capture carbon, along with a technoeconomic analysis. The complexity of this holistic approach has prevented researchers from publishing manuscripts or reports that look beyond tackling only one of the aspects mentioned above at a time. ${ }^{[48,115]}$

In the oxy-fuel processes, pure $\mathrm{O}_{2}$ instead of air is mixed with a fuel to combust it and obtain a clean stream of $\mathrm{CO}_{2}$ and $\mathrm{H}_{2} \mathrm{O} . \mathrm{CO}_{2}$ is then purified by solid filtering and $\mathrm{H}_{2} \mathrm{O}$ condensation. ${ }^{[119]}$ 
Pure $\mathrm{O}_{2}$ is a requirement and the cost of its separation from air represents one of the main costs. ${ }^{[110]}$ In this case, PI intervenes for the $\mathrm{O}_{2} / \mathrm{N}_{2}$ separation. A combination of cryogenic distillation with a $\mathrm{O}_{2} / \mathrm{N}_{2}$ permeable membrane (synergistic approach by function) where the membrane filters a stream of $\mathrm{O}_{2}$ - enriched air and the cryogenic distillation performs the final separation of $\mathrm{N}_{2}$ from $\mathrm{O}_{2}$ has the potential to decrease the cost. ${ }^{[120]}$

\section{PROCESS INTENSIFICATION AND MICROREACTORS}

Microreactor technology is another broad area covered by the literature on process intensification (PI). It is also linked to green chemistry, organic synthesis, and flow chemistry because translating processes from batch to continuous, possibly with intensified technology such as microreactors, represents a way of increasing energy and cost efficiency while minimizing waste. Microreactors above all demonstrate significant potential in the pharmaceutical industry. ${ }^{\text {[21] }}$ Microreactors can also be combined with other intensified technologies, such as microwave and ultrasound irradiation, photochemistry, inductive heating, heat exchangers etc., ${ }^{[20]}$ and electrochemical energy systems. ${ }^{[121]}$ For instance, a microreactor can be integrated with a residence-time module and a heat exchanger to be assembled in a unit composed of four microreactor elements (Figure 10, IMM for BASF). ${ }^{[122]}$

Microreactors, by now a mature technology that is being adopted worldwide, are typically miniaturized devices with a tubular reaction zone with ID $<\sim 1 \mathrm{~mm}$, most of which have submillimeter lateral dimensions and near-m illimeter flow channel diameters (often milli-reactors that have characteristic diameters larger than $1 \mathrm{~mm}$ are grouped with microreactors, but they do not share the same limitations, eg, clogging). ${ }^{[124]}$ Microreactor technology can profit from phenomena that are unique to microfluidic systems and include (a) laminar flow for single flows or defined multiphase flow patterns, (b) short diffusion paths for heat and mass transfer, (c) high surface-to- volume ratios , and (d) short paths for catalysts in suspension. ${ }^{[19]}$ 
Tanimu et $\mathrm{al}^{[125]}$ reviewed the latest developments in heterogeneous catalysis in microreactors. They describe designs where the catalyst either coats the microreactor walls (deposited through surface pretreatment or coating) or are inserted as packed beds or monoliths.

Examples of microreactor applications for chemical synthesis at the laboratory scale include: synthesis of organophosphate, ${ }^{[126]}$ propylene oxidation, ${ }^{[127]}$ hydrogenations, ${ }^{[128]}$ methanol steam reforming, ${ }^{[129]}$ and autothermal reforming, ${ }^{[130]}$ propane steam reforming, ${ }^{[131]}$ cross-coupling reaction, ${ }^{[132]}$ hydrogen peroxide production, ${ }^{[133,134]}$ and hydrogenations. ${ }^{[135-137]}$ Cherkasov et $\mathrm{al}^{[137]}$ reported the scale-up to $10 \mathrm{~kg} /$ day- $50 \mathrm{~kg} /$ day in capillary microreactors that were $0.53 \mathrm{~mm}$ and $1.6 \mathrm{~mm}$ in diameter for the hydrogenation of 2-methyl-butyn-2-ol.

Macchi et $\mathrm{al}^{[36]}$ recently reviewed microreactor design, technical features, challenges, and points of technological uncertainty within the scope of continuous synthesis of fine chemicals and pharmaceuticals, ranging from laboratory applications to commercial scale. They observe that despite the fact that the pharmaceutical industry is increasingly applying flow chemistry technologies to the synthesis of their products, the commercial application of intensified/miniaturized continuous flow technologies remain limited mostly due to lack of information and technology. As details on the reaction mechanism, the reaction rate parameters, heat of reaction(s), and transport rates are often unknown during molecular discovery, it is essential that chemists and chemical engineers work closely when designing microreactor devices. In fact, in continuous processing as an alternative to batch processing, experience with kinetics and fluid dynamics is required when both designing microreactors and scaling them up. Modestino et al ${ }^{[121]}$ highlighted strategies to scale-up energy micro-systems (but applicable to other technologies) by considering the dimensionality of the chemical processes. For instance, in ideally homogeneous chemical processes, where the reactor geometry does not affect the reaction (0D) microfluidic devices are not recommended. If a process is characterized by transport mechanisms on only one dimension (1D) (as is the case for electrochemical processes with parallel migration of charged species), then areal scaling is the best option. If the transport mechanisms occur in more than one dimension ( $\geq 2 \mathrm{D}$ ), as is the case of a heterogeneous catalytic 
reaction or a reaction mixture involving two-phases, then the only choice is parallelization (numbering up) (Figure 11). However, the authors also state that for microreactors numbering-up is challenging and other options should be sought if possible, ${ }^{[121]}$ in particular for multiphase systems.

The pharmaceutical industry faces obstacles when trying to innovate bench-scale and commercial-scale pharmaceuticals mass production through PI technologies, such as continuous reactors and microreactors, as they must comply with Pharmacopoeial standards and the any process change must be approved by the Food and Drug Administration (FDA). A group at MIT recently reported a fully automated, compact continuous flow synthesis laboratory system that can be configured to synthesize different molecules. This laboratory plant has already supplied hundreds to thousands of oral or topical doses of diphenhydramine hydrochloride, lidocaine hydrochloride, diazepam, and fluoxetine hydrochloride that meet United States Pharmacopeia standards. ${ }^{[138]}$

\section{PROCESS INTENSIFICATION AND KINETICS}

The macro-area on process intensification (PI) and kinetics (in blue in Figure 2) is the most multidisciplinary. It encompasses applications such as water treatment technologies, including liquid phase advanced oxidation processes, and biomass conversion. PI methods prevalently found in this macro-area are acoustic (ultrasound) and hydrodynamic cavitation.

Both ultrasound and hydrodynamic cavitation accelerate chemical reactions by either physical microscopic effects, thus improving micromixing and speeding extrinsic reaction kinetics (such in the case of biodiesel), or generating highly reactive radicals and speeding intrinsic (homogeneous) reaction kinetics (such as in the case of water treatment by advanced oxidation processes). ${ }^{[139,140]}$

Biodiesel is indeed one of the fields where the implementation of PI has been quite successful at the commercial scale and concerns hydrodynamic cavitation, ${ }^{[141]}$ controlled flow cavitation, and 
ultrasound. ${ }^{[142]}$ In biodiesel synthesis the two reagents are a triglyceride and an alcohol, usually methanol $\left(\mathrm{CH}_{3} \mathrm{OH}\right)$, which is only miscible at $5 \%$ at $65^{\circ} \mathrm{C}$ (transesterification temperature). ${ }^{[143]}$ Acoustic cavitation by ultrasound generates a variety of physical effects in the immiscible oil/alcohol mixture. Collapsing cavitation bubbles disrupt the phase boundary leading to the formation of a micro-emulsion of alcohol in oil. This translates in the increase in the interfacial area of exchange between the two reagents. These emulsions have a smaller droplet size and are more stable than those that conventional techniques generate. ${ }^{[144,145]}$ Ultrasound increases the process rate by at least two times in the presence of raw or waste oils. ${ }^{[146,147]}$ van Zwieten et $\mathrm{al}^{[148]}$ also demonstrated that an ultrasound bath in conjunction with a cavitation intensifying bag (CIB) (commercialized as BuBble bags by BuBclean, Enschede, The Netherlands), yielded hexane-sodium dodecyl sulphate (SDS) in water emulsion droplets from one to two orders of magnitude smaller than other emulsification techniques. The CIB concept can be seen as following the structure approach from the PI toolbox, where only the walls of the reactor were modified, but the rest of the equipment or process was left untouched.

Advanced oxidation processes (AOPs) are technologies used to degrade organic pollutants to $\mathrm{CO}_{2}$ and $\mathrm{H}_{2} \mathrm{O}$ in water through the generation of highly reactive hydroxyl radicals ('OH), which unselectively cleave and then oxidize contaminants. For this reason, ultrasound finds application mostly in water treatment. ${ }^{[149,150]}$ Ultrasound is an AOP since it breaks the O-H bond in water by generating hydrogen and hydroxyl radicals. ${ }^{[150]}$ Ultrasound can be combined with other AOPs in a synergistic approach to increase hydroxyl radicals concentrations. These approaches include photocatalysis, ${ }^{[151]}$ ozonation, ${ }^{[152]}$ Fenton reagent, ${ }^{[153]} \mathrm{H}_{2} \mathrm{O}_{2},{ }^{[154]}$ and microreactors. ${ }^{[139]}$ The largescale application of ultrasound to water treatment, whether alone or combined with another AOPs, is still limited because of the uncertainties related to its scale-up, as well as to the low efficiency of the other AOPs, in particular photocatalysis.

In hydrodynamic cavitation, cavities such as flow-through orifices, venturis, etc., produce pressure variations that create cavities that then implode, generating micromixing effects and radicals, similar to acoustic cavitation. ${ }^{[18]}$ Gogate ${ }^{[18]}$ also reports hydrodynamic cavitation as 40 
times more efficient than acoustic cavitation, and 160-400 times more efficient than conventional stirring in biodiesel synthesis. Water treatment is once again a popular field of application for hydrodynamic cavitation as a PI method. For instance, Angaji and Ghiaee ${ }^{[155]}$ designed a pilotscale hydrodynamic cavitation reactor with iron metal blades acting as a Fenton heterogeneous catalyst to degrade unsymmetrical dimethylhydrazine (UDMH). Similarly, Parsa and Zonouzian $^{[156]}$ designed and built a $30 \mathrm{~L}$ hydrodynamic cavitation reactor with plates made from scrap iron sheets to degrade Rhodamine B. Recently Ghorbani et al ${ }^{[157]}$ used cellulose nanofibers (CNF) to stabilize perfluorodroplets through Pickering emulsion and "hydrodynamic cavitation on chip.” Hydrodynamic cavitation can as well act as an AOP because of the hydroxyl radicals produced ${ }^{[158]}$ However, Arrojo and Benito ${ }^{[159]}$ point out that hydrodynamically generated hydroxyl radicals are more effective at oxidizing volatile and non-volatile hydrophobic substances as they only promote gas-phase and gas-liquid interactions, while it works poorly in liquid-phase oxidation.

\section{OUTLOOK}

The bibliometric map in Figure 2 displays the relationship between process intensification (PI) and related subjects between 1990-2019. This map is not static and will certainly evolve in the forthcoming years. New paradigms are becoming more and more important for the success of PI and to accelerate the adoption of intensified technologies at the commercial scale. We list the most promising technological archetypes hereinafter.

\subsection{Process intensification and electrification}

Renewable distributed energy generation (DEG) systems will play a key role in the near future for heat and power supply and to reduce our dependence on fossil fuels while reducing greenhouse gas (GHG) emissions. Renewable sources can indeed generate electricity, including solar, wind, geothermal, biomass, etc. Electricity appears as the natural way to power compact and modular chemical plants. It is also the way we power (catalytic) heated plates reactors, and 
heated microfluidic devices, as well as process intensification methods including ultrasound, microwaves, induction heaters, and plasma. Electrifying endothermic reactions or leveraging electricity to start up authothermal reactions, in particular micro-GtL or reforming plants, provide compact, mobile, and flexible reactors that do not rely on fuel supply. ${ }^{[160,161]}$ Moreover, concurrently with the multiplication of GtL plants, clean (electrolytic) $\mathrm{H}_{2}$ will become increasingly essential. This also applies to large-scale electrochemical processes, such as chloroalkali and aluminum production, which involve gas-evolving electrodes. ${ }^{[162]}$

\subsection{Process intensification and materials}

Since its origins, PI has been a relegated uniquely to intensifying equipment and methods. However, as the bibliometric map in Figure 2 displays with the subject catalysts at the centre, PI and materials are closely linked. Materials will become increasingly involved in the implementation of PI, in terms of their integration in both PI equipment and methods. The development of new materials or new uses for well-known materials offer unique opportunities in chemical reaction engineering by creating shape-selective geometries and well-defined pore structures to the nanometer scale. It is sufficient to think of zeolites, which have cavities that are both size and shape selective. Other properties such as super wettability, super hydrophobicity, magnetic and paramagnetic properties, magnetocaloric and metamaterials offer unique opportunities for PI. ${ }^{[46]}$ The developments in 3D printing technologies (eg, 3D printing catalytic modular unit)

may further accelerate the outcomes from the synergy between PI and materials.

\subsection{Process intensification and modularity}

Modular units, ie, micro-plants, reactors, distillation unit, and electrolytic stacks, will play a major role in the development of PI. Modular units possess several advantages, including increased efficiency by reducing process size and complexity and improving process safety and flexibility. Even though they are counterintuitive at first sight, modular units may also have 
lower capital and operating costs than full-size commercial units (see manufacturing experience below). For example, The United States Department of Energy (DOE) in 2016 funded the Manufacturing Innovation Institute on Modular Chemical Process Intensification for Clean Energy Manufacturing, which resulted in the creation of the Rapid Advancement in Process Intensification Deployment (RAPID) Institute. The DOE contributed \$70 million to RAPID, which also relies on the support of another \$85 million from partners over five years. One of the main aims of RAPID is to "leverage approaches to modular chemical process intensification (MCPI) — such as combining multiple process steps such as mixing, reaction, and separation into single more complex and intensified processes — with the goal of improving productivity and efficiency, cutting operating costs, and reducing waste.”[163]

\subsection{Process intensification and experience manufacture}

Intensification strategies tend to be more feasible at certain length and time scales, e.g., at smaller and manageable scales by manufacturing modular units (Figure 3). To meet economies of scales, modular units must adhere to the notion of numbering-up (scaling down and then scaling out) (see Figure 10). Numbering-up is often accompanied by a financial model that does not always appeal to investors. This model requires solid financial prognostics to reduce uncertainties around the fate of their assets. Numbering-up models appeal to the concept of experience, ie, the reduction of costs that includes learning and building new and more numerous facilities of the same type. ${ }^{[164]}$ As outlined in Section 5.1, traditional economic models either scale-up or scale-down the investment (C) for a given plant size (Q) with a power law, whereby the exponent $\alpha$ depends on the characteristic unit size (n). New financial models to scale up by numbering-up should become mainstream. Learning elasticity $\beta$, which still correlates with the characteristic unit size $\mathrm{n}$, is among the options. ${ }^{[102]}$

\subsection{Process intensification and artificial intelligence (Industry 4.0)}


While simultaneously aiming at maximizing mass, heat, and momentum transfer, processintensified reactors and elements often possess complex structures and geometry such as static mixers, microchannels reactors, as well as multi-phase reactors, such as rotating packed and fluidized beds. The combination of experimental data with advanced visual aids and/or computational fluid dynamics (CFD) modelling is key to understanding micromixing scales and calculate the energy density of the intensified process. ${ }^{[48]}$ In this context we expect that the Industry 4.0 will accelerate the availability of new digital platforms for fast and reliable real-time data sharing. Industry 4.0 may not only accelerate the adoption of PI technologies in the market but also make the engineering, procurement and construction companies (EPC) workflow more efficient when it comes to the fabrication and engineering of modular units. ${ }^{[12,81]}$ This will be possible as it advocates for digitizing both manufacturing (smart manufacturing) and business processes, which not only promise to increase manufacturing productivity but also tackle resource efficiency and waste reduction.

\subsection{Quantifying process intensification}

We believe that the scientific evidence of the benefits of PI is abundant, but the proven economic advantages of PI at the commercial scale are more difficult to find. The metrics to compare traditional technologies with PI technologies, which can aid in defining when to intensify a process, are still in their early stages. However, some examples have started appearing in the literature. For instance, Fernandez Rivas et al ${ }^{[78]}$ proposed a simple method to calculate the intensification factor (IF) as $I F=\left(\frac{F_{b}}{F_{a}}\right)^{d}$, where $\mathrm{Fb}_{\mathrm{b}}$ and $\mathrm{F}_{\mathrm{a}}$ are the input factors before and after intensification, respectively (eg, $\mathrm{F}=$ temperature, residence time, selectivity, etc.), and $d$ determines whether a decrease or increase in $\mathrm{F}$ is desired. If more elements are intensified in the same process, the total intensification will be $I F_{\text {tot }}=\sum_{i=1}^{p}\left(I F_{i}\right)^{c_{i}}$, where ci is the level of importance of the IF (eg, avoided environmental impacts), which must be agreed upon. For example, an intensified one-step reversed osmosis (OSRO) drinking water treatment plant gives 
(IF) total $=18.56$ compared to a traditional water treatment train with seven steps (Figure 12). ${ }^{[78]}$ Clearly, methods to calculate IFs or other PI metrics may attract interest from investors and companies, but they only serve as a complement to techno-economic analysis (TEA) and life cycle analysis (LCA), which ultimately determine the appeal and sustainability of a process.

\subsection{Barriers to commercialization}

We recently identified several barriers to the commercialization and adoption of PI by industry: (a) lack of success stories, (b) convenience of unit operations-oriented disciplines, (c) conservativism in upper management/risk aversion, (d) supply chain fragility, and (e) perceived scalability issues. Education on PI was proposed as a means of overcoming these barriers and practical examples about how to do so were provided. ${ }^{[12,13]}$

To conclude, the chemical engineering community can expect new developments and opportunities facilitated by PI. However, it is important to focus on reliable information sources and try not to raise expectations too much; not all needs can be intensified.

\section{ACKNOWLEDGEMENTS}

This research was undertaken, in part, thanks to funding from the Canada Research Chairs program. David Fernandez-Rivas’ participation was supported by the Netherlands Centre for Multiscale Catalytic Energy Conversion (MCEC), an NWO Gravitation program funded by the Ministry of Education, Culture and Science of the Government of the Netherlands.

We are grateful to Prof. Gregory Patience (Polytechinique Montréal, Department of Chemical Engineering) for providing the bibliometric data and map, as well as Claudio Boffito for the graphical support.

\section{Declaration of Interests}


David Fernandez-Rivas is the founder and scientific advisor of BuBclean VOF, a start-up company commercializing ultrasonic cleaning solutions via process intensification.

\section{REFERENCES}

[1] W. J. Podbielniak, "US patent 2,004,011," 1935.

[2] P. Rao, Indian Chem. Eng. (2015), 57(3), 1.

[3] K. C. D. Hickman, "UK Patent 482880," (1936).

[4] D. Reay, C. Ramshaw, A. Harvey, Process Intensification, 1st ed., Elsevier (2008).

[5] D. Reay, C. Ramshaw, A. Harvey, Process Intensification, 2nd ed., Elsevier, (2015).

[6] C. Ramshaw, presented at 1st International Conference on Process Intensification in the Chemical Industry, Antwerp, December 1995.

[7] A. Stankiewicz, J. A. Moulijn, Chem. Eng. Prog. 2000, 1, 22.

[8] C. Tsouris and J. V. Porcelli, Chem. Eng. Prog., 2003, 99(10), 50.

[9] European Federation of Chemical Engineering, European Roadmap for Porcess Inetsnification, https://efce.info/efce_media/-p-531-EGOTEC06098dab96249739438513dad9100227.pdf (accessed: January 2020)".

[10] S. Becht, R. Franke, A. Geisselman, H. Hahn, Chem. Eng. Process., 2009, 48(1), 329.

[11] T. Van Gerven, A. Stankiewicz, Ind. Eng. Chem. Res. 2009, 48(5), 2465.

[12] D. Fernandez Rivas, D. C. Boffito, J. Faria-Albanese, J. Glassey, N. Afraz, H. Akse, K. V. K. Boodhoo, R. Bos, J. Cantin, W. Chiang, J. Commenge, J. Dubois, F. Galli, J. H. J. 
Gueneau de Mussy, S. Kalra aet al., , Educ. Chem. Eng. 2020, 10.1016/j.ece.2020.04.003, 2020.

[13] D. Fernandez Rivas, B. D. C., J. Cantin, J. Glassey, J. Faria-Albanese, N. Afraz, H. Akse, K. V. K. Boodhoo, R. Bos, Y. W. Chiang, J. M. Commenge, J. L. Dubois, F. Galli, J. P. Gueneau de Mussy, J. Harmse, S. Karlra et al., Educ. Chem. Eng., 2020, 10.1016/j.ece.2020.05.001, 2020.

[14] The Chemistry Industry Association of Canada, About Resposible care, https://canadianchemistry.ca/responsible-care/about-responsible-care/\#, Accessed on 2020, May 20th, (2020).

[15] R. Thakur, C. Vial, K. Nigam, E. Nauman, G. Djelveh, Chem. Eng. Res. Des. 2003, 81(7), 787.

[16] A. Ghanem, Y. Lemenand, D. Della Valle, H. Peerhossaini, Chem. Eng. Res. Des., 2013, 92(2), 205.

[17] S. Jegatheeswaran, F. Ein-Mozaffari, J. Wu, Rev. Chem. Eng. 2020, 36(3), 423.

[18] P. Gogate, Chem. Eng. Proc. 2008, 47(4), 515.

[19] V. Hessel, D. Kralisch, N. Kockmann, T. Noël, Q. Wang, ChemSusChem, 2013, 6, 746.

[20] R. Porta, M. Benaglia, A. Puglisi, Org. Process Res. Dev. 2016, 20, 1.

[21] L. Vaccaro, D. Lanari, A. Marrocchi, G. Strappaveccia, Green Chem. 2014, 16, 3680.

[22] I. Baxendale, J. Hayward, S. Ley, Comb. Chem. High. Throughput Screen, 2007, 10(10), 802. 
[23] N. Kockmann and D. Roberge, Chem. Eng. Technol., 2009, 32(11), 1682.

[24] C. Sambiagio, T. Noel, Trends Chem., 2020, 2(2), 92.

[25] P. Bernardo, E. Drioli, G. Golemme, Ind. Eng. Chem. Res., 2009, 48(10), 4638.

[26] F. Gallucci, E. Fernandez, P. Corengi, M. van Sint Annaland, Chem. Eng. Sci., 2013, 92, 40.

[27] P. Luis, T. Van Gerven, B. Van der Bruggen, Prog. Energy. Combust Sci., 2012, 38(3), 419.

[28] A. A. Kiss, J. J. Pragt, C. J. G. van Strien, Chem. Eng. Comm, 2009, 196, 1366.

[29] A. Kiss, J. Chem. Technol. Biotechnol. 2014, 89(4), 479.

[30] A. Gavriilidis, P. Angeli, C. E., K. Yeong, Y. Wan, Chem. Eng. Res. Des., 2002, 80(1), 3.

[31] M. Kashid, L. Kiwi-Minsker, Microstructured Reactors for Multiphase Reactions: State of the Art, Ind. Eng. Chem. Res., 2009, 48(14), 6465.

[32] M. Oelgemöller, O. Shvydkiv, Recent Advances in Microflow Photochemistry, Molecules, 2011, 16, 7522.

[33] R. Wohlgemuth, I. Plazl, P. Žnidaršič-Plazl, K. Gernaey and J. Woodley, Trends in Biotechnol. 2015, 33(5), 302.

[34] X. Yao, Y. Zhang, L. Du, J. Liu, J. Yao, Renew. Sust. Energ. Rev. 2015, 47, 519.

[35] N. Herrmann, P. Neubauer, M. Birkholz, Biomicrofluidics 2019, 13(6), 061501.

[36] A. Macchi, P. Plouffe, G. Patience, D. Roberge, Can. J. Chem. Eng., 2019, 97, 2578. 
[37] J. Singh, A. Montesinos-Castellanos, K. Nigam, Ind. Eng, Chem. Res.2019, 58(31), 13819.

[38] M. Gawande, S. Shelke, R. Zboril, R. Varma, Acc. Chem. Res. 2014, 47(4), 1338.

[39] H. Li, Z. Zhao, C. Xiouras, G. Stefanidis, L. X. and X. Gao, Renew. Sust. Energ. Rev., 2019, 114, 109316.

[40] V. Sutkar, P. Gogate, Chem. Eng. J. 2009, 155(1), 26

[41] E. Rokhina, P. Lens, J. Virkutyte, Trends. Biotechnol. 2009, 27(5), 298.

[42] Z. Dong, C. Delacour, K. Mc Carogher, A. Udepurkar, S. Kuhn, Materials 2020, 13(2), 344.

[43] S. Sitter, Q. Chen, I. Grossmann, Curr. Opin. Chem. Eng. 2019, $25,87$.

[44] Y. Tian, E. Pistikopoulos, Curr. Opin. Chem. Eng. 2019, I, 101.

[45] S. Demirel, J. Li and F. Hasan, Curr. Opin. Chem. Eng. 2019, 25, 108.

[46] A. Stankiewicz, P. Yan, Ind. Eng. Chem. Res. 2019, 58, 22, 9212.

[47] A. Stankiewicz, Chem. Eng. Res. Des. 2006, I, 511.

[48] D. C. Boffito, T. Van Gerven, in Reference Module in Chemistry, Molecular Sciences and Chemical Engineering (Ed: J. Reedijk, Elsevier, Waltham 2019, p. 1.

[49] J. F. Chen, presented at the GPE-EPIC, Venice, June 2000

[50] M. Stec, P. Synowiec, Can. J. Chem. Eng., 2017, 95, 2156.

[51] M. Stec, P. Synowiec, Can. J. Chem. Eng., 2017, 95, 2410. 
[52] M. Stec, P. Synowiec, Can. J. Chem. Eng., 2019, 97, 995.

[53] Z. Wang, T. Yang, Z. Liu, S. Wang, Y. Gao, M. Wu, Chem. Eng. Process. 2019, 139, 78.

[54] D. Wenzel, A. Górak, Chem. Eng. J., 2018, 345, 292.

[55] L. Wu, W. Geng, L. Gao, J. Chen, H. Zhao, Energ. Fuel 2017, 31, 13400.

[56] M. Sheng, B. Sun, F. Zhang, G. Chu, L. Zhang, C. Liu, Energ. Fuel 2016, 30, 4215.

[57] H. Zou, M. Sheng, X. D. Sun, M. Arowo, Y. Luo, L. C. Zhang et al., Fuel 2017, $204,47$.

[58] J. Xu, C. Liu, M. Wang, L. Shao, L. Deng, K. Nie, F. Wang, Bioresour. Technol. 2017, 224, 292.

[59] B. Sun, L. Zhang, Z. Weng, L. Zhang, G. Chu, H. Zou, J. Chen, Chem. Eng. Process, 2017, 119, 93.

[60] P. Oxley, C. Brechtelsbauer, F. Ricard, N. Lewis, C. Ramshaw, Ind. Eng. Chem. Res. 2000, 39(7), 2175.

[61] K. V. K. Boodhoo and R. J. Jachuck, Green Chem. 2000), 2, 235.

[62] S. D. Pask, Z. Cai, H. Mack, L. Marc, O. Nuyken, Macromol. React. Eng. 2013, 7, 98.

[63] H. C. Yatmaz, C. Wallis, C. Howarth, Chemosphere 2001, 42, 397.

[64] J. McDonough, A. Phan, A. Harvey, Chem. Eng. J. 2015, 265, 110.

[65] N. Ni, M. Mackley, A. Harvey, P. Stonestreet, M. Baird, N. Rao, Chem. Eng. Res. Des. 2003, 81(3), 373.

[66] J. McDonough, M. Oates, R. Law,A. Harvey, Chem. Eng. J. 2019 , 361, 508. 
[67] F. González-Juárez, D. Martínez, R. Herrero-Martín, J. Solano, presented at the 12th International Conference on Heat Transfer, Fluid Mechanics and Thermodynamics, Malaga, July 2016.

[68] V. Eze, A. Phan, C. Pirez, A. Harvey, A. Lee, K. Wilson, Cat. Sci. Technol. 2013, 3, 2373.

[69] V. Eze, A. Harvey, Chem., Eng. J. 2018, 347(1), 4.

[70] A. Phan, A. Harvey, V. Eze, Chem. Eng. Technol. 2012, 35, 1214.

[71] M. Soufi, B. Ghobadian, S. Mousavi, G. Najafi, Aubin, Energy 2019, I, 116266.

[72] R. Rasdi, A. Phan, A. Harvey, Chem. Eng. J. 2013, I, 282.

[73] B. Wilson, D. Sherrington, X. Ni, Ind. Eng. Chem. Res. 2005, 44, 8663.

[74] P. Gao, W. Han Ching, M. Herrmann, C. Kwong Chan, P.L. Yue, Chem. Eng. Sci. 2003, $58,1013$.

[75] C. Brown, X. Ni, Cryst. Growth Des.2011 , 11, 719.

[76] T. McGlone, N. Briggs, C. Clark, C. Brown, J. Sefcik, A. Florence, Org. Process Res. Dev. 2015, 19(9), 1186.

[77] N. Reis, R. Pereira, A. Vicente, J. Teixeira, Ind. Eng. Chem. Res. 2008, 47(19), 7190.

[78] D. Fernandez-Rivas, E. Castro-Hernández, A. L. Villanueva Perales, W. van der Meer, Chem. Eng. Process. 2018, 123, 221.

[79] W. Poe, S. Mokhatab, Modeling, Control, and Optimization of Natural Gas Processing Plants, 1, Elsevier, Cambridge 2017, p. 1. 
[80] A. Marchetti, G. François, T. Faulwasser, D. Bonvin, Processes 2016, 4, 55.

[81] R. Aglave, J. Lusty, J. Nixon, Chem. Eng. Progr. 2019, March, 45.

[82] W. Marquardt, L. von Wedel, B. Bayer, presented at the $5^{\text {th }}$ International conference, Foundations of computer-aided process design, Breckenridge, July 1999.

[83] A. Moulijn, Stankiewicz, J. Grievink, A. Górak, Comput. Chem. Eng. 2008, 32 , 3.

[84] J. Bergerson, A. Brandt, J. Cresko, M. Carbajales-Dale, H. L. M acLean, H. M atthews, S. McCoy, M. McManus, S. Miller, W. Morrow III, I. Posen, T. Seager, T. Skone, S. Sleep, J. Ind. Ecol. 2020, 24, 11.

[85] K. Motazedi, J. Abella, J. Bergerson, Environ. Sci. Technol. 2017, 51(3), 1918.

[86] D. Pacheco, J. Bergerson, A. Alvarez-Majmutov, J. Chen and H. MacLean, Environ. Sci. Technol. 2016, 50(24), 13574.

[87] S. Sleep, I. Laurenzi, J. Bergerson, H. MacLean, Environ. Sci. Tehnol. 2018, 52(20), 11941.

[88] I. Laurenzia, J. Bergerson and K. Motazedi, PNAS 2016, 113, 48.

[89] I. Dejanović, L. Matijašević, Ž. Olujić, Chem. Eng. Process. 2010, 49(6), 559.

[90] Ž. Olujić, M. Jödecke, A. Shilkin, G. Schuch, B. Kaibel, Chem. Eng. Process. 2009, 48, 1089.

[91] J. Harmsen, Chem. Eng. Process. 2010, 9(1), 70.

[92] G. Tech., https://papers.gtctech.com/whitepaper/top-dividing-wall-column-technology-anovel-approach/, Accessed on 2020 May, 20th. 
[93] M. Bhargava, Chem. Eng. Prog., 2019, March, 30.

[94] A. A. Backhaus., US Industrial Alcohol Co., inv., US 1403224, 1922.

[95] M. Kumar, A. K. Mathew, Can. J. Chem. Eng. 2017, 95, 331.

[96] G. Hamsen, Chem. Eng. Process. 2007, 46, 774.

[97] Q. K. Le, I. J. Halvorsen, O. Pajalic, S. Kogestad, Chem. Eng. Res. Des. 2015, 99, 111.

[98] B. Suphanit, A. Bischert, P. Narataruksa, Energy 2007, 32(11), 2121.

[99] H. S. Fogler, Elements of Chemical Reaction Engineering, 5th Ed., Wiley, Kendallville 2016, p 439.

[100] A. Lind, Ø. Vistad, M. F. Sunding, K. A. Andreassen, J. Hafizovic Cavka, C. A. Grande, Mater. Design, 2020, 197, 108377.

[101] J. T. Richardson, Principles of Catalyst Development, Springer, New York 1989, p.1.

[102] G.S. Patience, D.C. Boffito, Adv. Manuf. Process. 2020,_2, e10039.

[103] Z. Ma, P. Perreault, D. C. Pelegrin, D. C. Boffito, G. S. Patience, Chem. Eng. J. 2020, 380(15), 122470.

[104] G. S. Patience, D. C. Boffito, Can. J. Chem. Eng. 2016, 94, 605.

[105] C. Trevisanut, S. Jazayeri, S. Bonkane, C. Neagoe, A. Mohamadalizadeh, D. Boffito, C.L. Bianchi, C. Visconti, L. Lietti, N. Abatzoglou, L. Frost, J. Lerou, W. Green, G. Patience, Can. J. Chem. Eng. 2016), 94, 613. 
[106] C. Neagoe, D. C. Boffito, Z. Ma, C. Trevisanut, G.S. Patience, Catal. Today, 2016, 270, 43.

[107] Z. Ma, P. Ouzilleau, C. Trevisanut, C. Neagoe, S. Lotfi, D. Boffito, G. Patience, Can. J. Chem. Eng., 2016, 94, 642.

[108] O. Onel, A. Niziolek, H. Butcher, B. A. Wilhite, C. A. Floudas, Comput. Chem. Eng., 2017, 105, 276.

[109] B. Hallmark, J. Parra-Ga rrido, A. Murdoch, I. Salmon, C. Hodrien, Can. J. Chem. Eng., 2017, 95, 1023.

[110] J.C.M. Pires, F.G. Martins, M.C.M. Alvim-Ferraz, M.Simões, Chem. Eng. Res. Des. 2011, 89(9), 1446.

[111] M. Abdollahi, J. Yu, H. T. Hwang, P. K. Liu, R. Ciora, M. Sahimi, T. T. Tsotsis, Ind. Eng. Chem. Res. 2010), 49(21), 10986.

[112] U. Ahmed, U. Zahid, Y. S. Jeong, C. J. Lee, C. Han, Chem. Eng. Process. 2016, 101, 72.

[113] H. Chen, M. Cao, L. Zhao, R.J. Ciora Jr., P.K.T. Liu, V.I. Manousiouthakis, T.T. Tsotsis*, Experimental Study of an Intensified Water-Gas Shift Reaction Process Using a Membrane Reactor/Adsorptive Reactor Sequence, Ind. Eng. Chem. Res. 2018, I, 1365013660.

[114] P. Pichardo, S. Karagöz, V. Manousiouthakis, T. Tsotsis and R. Ciora, Ind. Eng. Chem. Res. 2020, 59(6), 24, 30.

[115] M. Wang, A. S. Joel, C. Ramshaw, D. Eimer, N. M. Musa, Appl. Energy, 2015, 158, 275. 
[116] M. R. M. Abu-Zahra, J. P. M. Niederer, P. H. M. Feron and G. F. Versteeg, Int. J. Greenh. Gas Control. 2007, 1, 135.

[117] A. Joel, M. Wang, C. Ramshaw, E. Oko, Appl. Energy 2017, 1, 11.

[118] F. Zarei, M. Rahimi, R. Razavi, A. Baghban, J. Clean. Prod. 2019, 211, 953.

[119] R. Ahmed, G. Liu, B. Yousafa, Q. Abbasa, H. Ullah, M. Ali, , J. Clean. Prod. 2020, 242, 118409.

[120] T. Burdyny, H. Struchtrup, Energy 2020, 35(5), 1884.

[121] M. A. Modestino, D. Fernandez Rivas, S. M. H. Hashemi, J. G. E. Gardeniers and D. Psaltisa, Energy Environ. Sci., 2016, 9, 3381.

[122] K. Jähnisch, V. Hessel, H. Löwe, M. Baerns, Angew. 2004, I, 406.

[123] Proceedings of the First International Conference on Microreaction Technology, (Ed: E. Wolfgang), Springer, Berlin 1998.

[124] S. G. Kandlikar, M. R. King, Heat Transfer and Fluid Flow in Minichannels and Microchannels, 2nd ed, Elsevier, Oxford 2014, p1.

[125] A. Tanimu, S. Jaenicke, K. Alhooshani, Chem. Eng. J. 2017, 327, 792.

[126] Y. Zhang, J. Li, Y. Jin, M. Chen, Y. Wang, Can. J. Chem. Eng. 2020, 98(5), 1139.

[127] Y. H. Yuan, X. G. Zhou, W. Wu, Y. R. Zhang, W. K. Yuan, L. Luo, Catal. Today 2005, 105, 544.

[128] S. Schubert, R. Schellen, L. Mleczko, S. Laue, Bayer Technology Services GmBH, US Patent Application 2009/0093655, 2009. 
[129] S. M. Hwang, O. J. Kwon, J. J. Kim, Appl. Catal. A 2007, 316, 83 .

[130] C. Horny, A. Renken, L. Kiwi-Minsker, Catal. Today 2007, 120, 45.

[131] G. Kolb, R. Zapf, V. Hessel, H. Löwe, Appl. Catal. A 2004, 277, 155.

[132] U. Kunz, H. Schönfeld, W. Solodenko, G. Jas, A. Kirschning, Ind. Eng. Chem. Res. 2005, 44, 8458.

[133] H. P. .. L. Gemoets, Y. Su, M. Shang, H. V., R. Luque, T. Noel, Chem. Soc. Rev. 2016, 45, 83.

[134] G. Wu, G. L. Brett, E. Cao, E. P. Constantinou, S. Kuhn, G. J. Hutchings, D. Bethell, A. Gavriilidis, Catal. Sci. Technol., 2016, 6, 4749.

[135] L. N. Protasova, E. V. Rebrov, H. E. Skelton, A. E. H. Wheatley, J. C. Schouten, Appl. Catal. A 2011, 399, 12.

[136] N. Cherkasov, A. O. Ibhadon, E. V. Rebro, Appl. Catal. A Gen., 2016, 515, 108.

[137] N. Cherkasov, M. Al-Rawashdeh, A. O. Ibhadon, E. V. Rebrov, Catal. Today 2016, 273, 25.

[138] A. Adamo, R. L. Beingessner, M. Behnam, J. Chen, T. F. Jamison, K. F. Jensen, J. C. Monbaliu, A. C. Myerson, E. M. Revalor, D. R. Snead, T. Stelzer, N. Weeranoppanant, S. Y. Wong, P. Zhang, Science 2016, 352, 61.

[139] D. Fernandez Rivas, P. Cintas, H.J.G.E. Gardeniers, Chem. Commun. 2012, 48, 10935.

[140] D. Fernandez Rivas, S. Kuhn, Top. Curr. Chem. 2016, 374, 70.

[141] P. R. Gogate, A. B. Pandit, Rev. Chem. Eng.2001, 17, 1. 
[142] Hilesher, https://www.hielscher.com/biodiesel_transesterification_01.htm, Accessed on 2020 January $27^{\text {th }}$.

[143] C. Pirola, F. Manenti, F. Galli, C. Bianchi, D. Boffito, M. Corbetta, Chem. Eng. Trans. 2014, 37, 553.

[144] D. C. Boffito, C. Pirola, C. L. Bianchi, G. Cerrato, S. Morandi and A. Muthupandian, in Producing Fuels and Fine Chemicals from Biomass Using Nanomaterials, (Eds: A. Balu, R. Luque), CRC Press, Boca Raton 2013, p. 137.

[145] D. C. Boffito, S. Mansi, J. Leveque, C. Pirola, C. L. Bianchi, G. S. Patience, ACS Sust. Chem. Eng. 2013, 1(11), 1432.

[146] D. C. Boffito, E. Martinez-Guerra, V. P. G. Gude, in Handbook of Ultrasonics Sonochemistry, (Ed: M. Ashokkumar), Springer, Singapore 2015, p. $1 .$.

[147] D. C. Boffito, F. Galli, C. Pirola, C. L. Bianchi, G. S. Patience, Utrason. Sonochem. 2014, 21(6), 1969.

[148] R. van Zwieten, B. Verhaagen, K. Schroën, D. Fernández Rivas, Ultrason. Sonochem. 2017, 36, 446.

[149] Z. Khani, D. Schieppati, C. L. Bianchi, D. C. Boffito, Catalysts 2019, 9(11), 949.

[150] N. N. Mahamuni, Y. G. Adewuyi,, Ultrason. Sonochem. 2010, 17(6), 990.

[151] D. Schieppati, F. Galli, M. L. Peyot, V. Yargeau, C. L. Bianchi, D. C. Boffito, Ultrason. Sonochem. 2019, 54, 302.

[152] H. Destaillats, A. J. Colussi, J. M. Joseph, M. R. Hoffmann, J. Phys. Chem. A. 2003, 104, 8930. 
[153] B. Neppolian, H. Jung, H. Choi, J. H. Lee, J. W. Kang, Water Res. 2002, 36, 4699.

[154] F. J. Beltrtan, Ozone Reaction Kinetics for Water and Waste Water Systems, Lewis Publishers, CRC Press, Boca Raton, 2003, p.1

[155] M. Angaji, R. Ghiaee, Ultrason. Sonochem. 2015, 23, 257.

[156] J. Parsa, S. Zonouzian, Ultrason. Sonochem. 2013, 20(6), 1442.

[157] M. Ghorbani, A. S. Aghdam, M. T. Gevari, A. Koşar, F. Ç. Cebeci, D. Grishenkov, A. J. Svagan, Chem. Eng. J. 2020, 382, 122809.

[158] K. S. Suslick, M. M. Mdleleni and J. T. Ries, J. Am. Chem. Soc. 1997, 119(39), 9303.

[159] S. Arrojo, Y. Benito, Ultrason. Sonochem. 2008, 15(3), 203.

[160] S. T. Wismann, J. S. Engbæk, S. B. Vendelbo, W. L. Eriksen, C. Frandsen, P. M. Mortensen, I. Chorkendorff, Ind. Eng. Chem. Res. 2019, 58(51), 23380.

[161] S. T. Wismann, J. S. Engbæk, S. B. Vendelbo, F. B. Bendixen, W. L. Eriksen, K. Aasberg-Petersen, C. Frandsen, I. Chorkendorff, M. Mortensen, Science 2019, 364(6442), 756.

[162] A. Angulo, P. van der Linde, H. Gardeniers, M. Modestino, D. Fernandez Rivas, Influence of Bubbles on the Energy Conversion Efficiency of Electrochemical Reactors, Joule 2020, 4(3), 555

[163] https://www.aiche.org/rapid, Accessed on January $15^{\text {th }} 2020$. 
[164] B. Henderson, Perspectives on Experience, The Boston Consulting Group, Boston 1972, p.1 


\section{Figure captions}

FIGURE 1 The number of papers in the Web of Science Core Collection with process intensification as a topic increased exponentially since 1992 with $\mathrm{N}_{\text {art }}=10^{(0.0791 \mathrm{y}-157)}$ for a total number of articles $\mathrm{N}_{\mathrm{art}}=3480$

FIGURE 2 Bibliometric network presenting the frequency of keywords and their relationships to process intensification. The VOSviewer generated the image from data indexed by Web of Science (WoS) as of 23 January 2020. The size of the circles and text are proportional to the number of occurrences in the keywords. The largest circle is in the map is mass transfer (389), followed by design (296), reactor (269), optimization (251), and mass transfer (110). The smallest circles correspond to 35 occurrences (eg, two-phase flow, residence time distribution, etc.). Colors group topics that are closely related and the lines connect subjects

FIGURE 3 Mass and heat transfer capacities in common process-intensifying equipment. Reproduced with permissions from Chen ${ }^{[49]}$

FIGURE 4 Independence and synergy of process intensification (PI) and process system engineering (PSE). Adapted from Moulijn et al ${ }^{[83]}$

FIGURE 5 Dividing-wall column (DWC) configurations: A, middle; B, top; C, bottom. Reproduced with permissions from Le et $\mathrm{al}^{[97]}$

FIGURE 6 The relationship between process intensification and heterogeneous catalysis. Reproduced with permission from Boffito and Van Gerven ${ }^{[48]}$ 
FIGURE 7 Process flow diagram of a gas-to-liquid plant with tandem methane catalytic partial oxidation (CPOX) and Fischer-Tropsch for clean fuels production. Reproduced with permission from Trevisanut et al ${ }^{[105]}$

FIGURE 8 Proposed areas of process intensification (PI) for carbon capture and storage (CCS) technologies, most by synergistic PI

FIGURE 9 A membrane reactor in tandem with an adsorptive reactor (MR-AR) in integrated gasification and combined cycle (IGCC) power plants produce more power and have lower capital cost than traditional precombustion carbon capture systems. Published with permission from Pichardo et al ${ }^{[114]}$

FIGURE 10 Microstructured reactor with mixer, residence-time module, and heat exchanger (manufactured by Fraunhofer IMM for BASF for the highly exothermic synthesis of a vitamin precursor). ${ }^{[122,123]}$ Reproduced with permission from Jähnisch et al ${ }^{[122]}$

FIGURE 11 Scaling strategies for increasing the throughput of microfluidic reactors. E-chem = electrochemical reaction. Reproduced with permission from The Royal Society of Chemistry ${ }^{[121]}$

FIGURE 12 A, traditional water treatment train with seven steps (filtration, aeration, sand filtration, softening, second aeration, second sand filter step, active carbon filter, and a final ultraviolet disinfection step; B, novel process intensification called one-step reverse osmosis (OSRO) with only four steps. Reproduced with permission from Fernandez-Rivas et al ${ }^{[78]}$ 


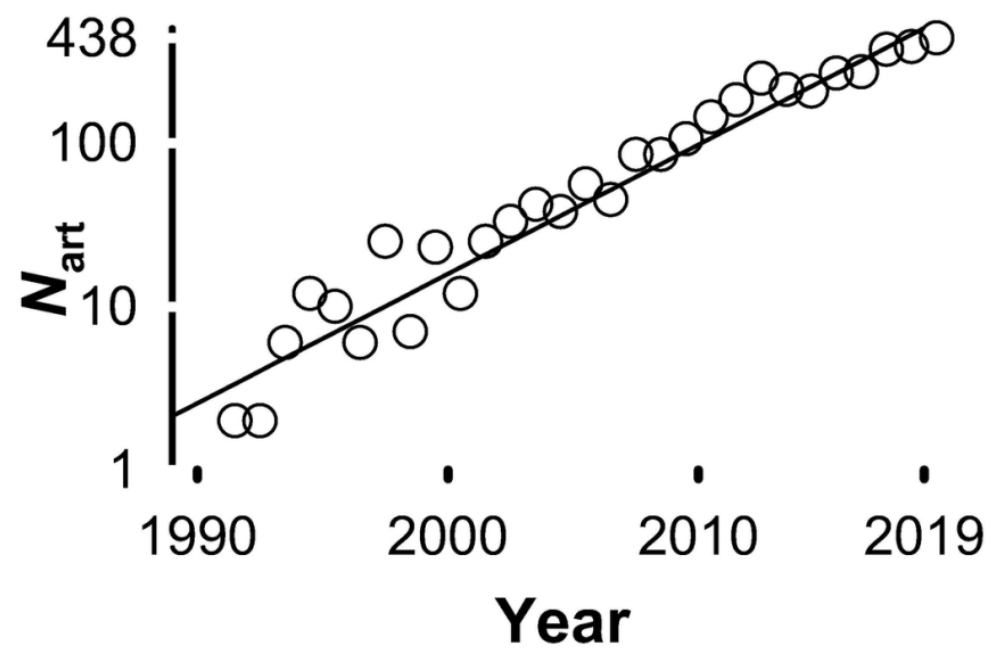

FIGURE 1 The number of papers in the Web of Science Core collection with "Process Intensification" as a topic increased exponentially since 1992 with Nart $=10(0.0791$ y -157$)$ for a total number articles Nart $=3$ 480 


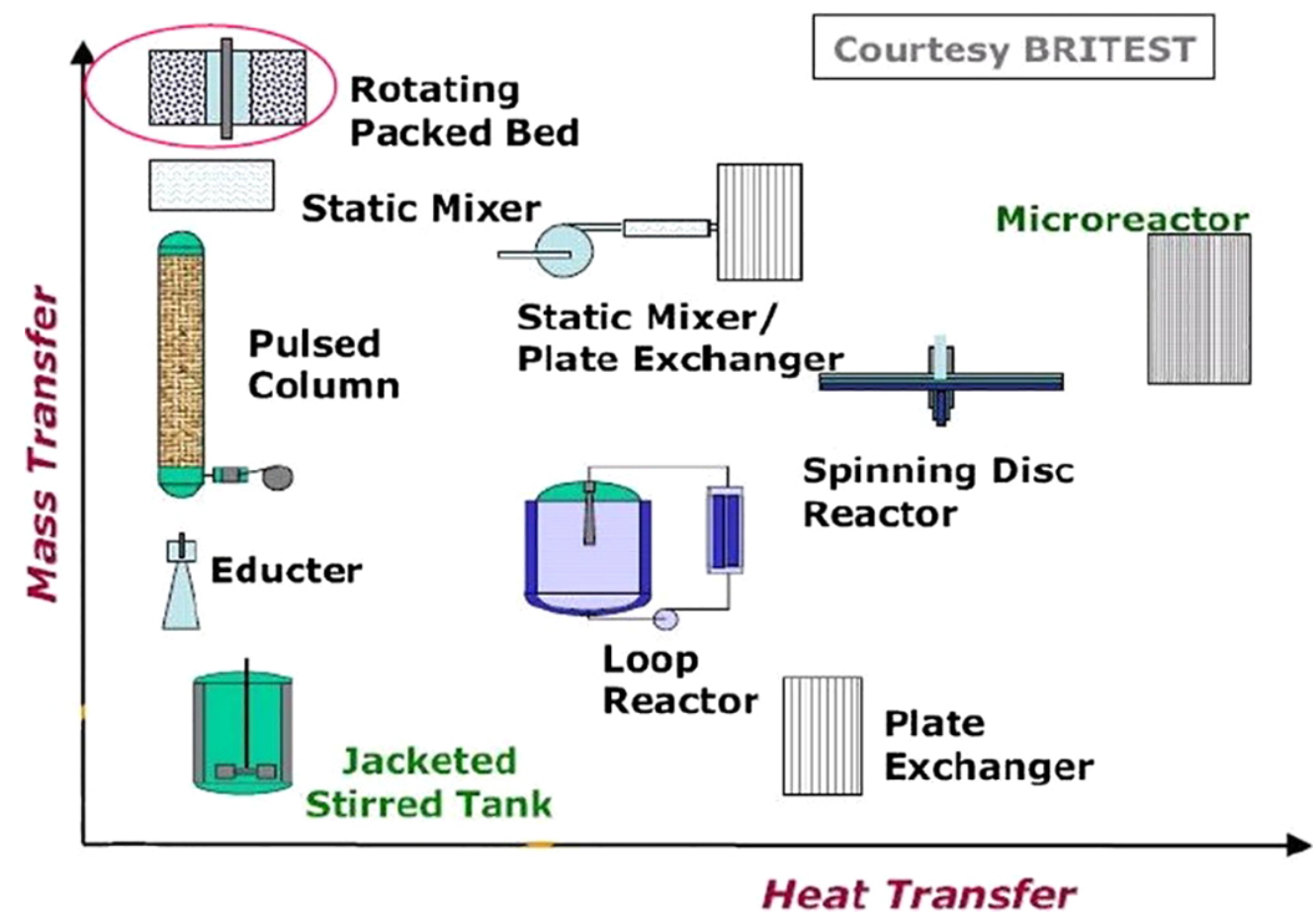

FIGURE 3 Mass and heat transfer capacities in common process-intensifying equipment. Reproduced with permissions from Chen[49] 


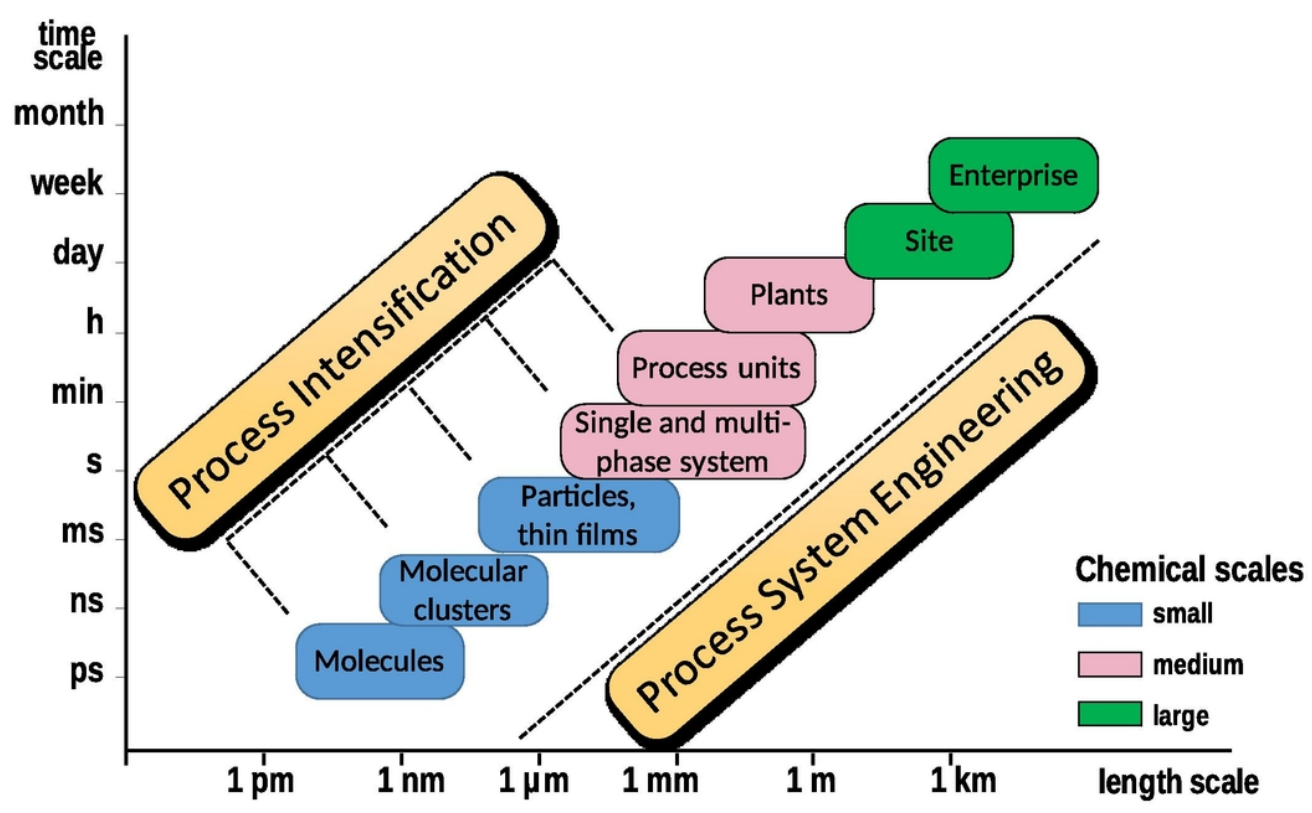

FIGURE 4 Independence and synergy of process intensification (PI) and process system engineering (PSE) Adapted from Moulijn et al[83] 

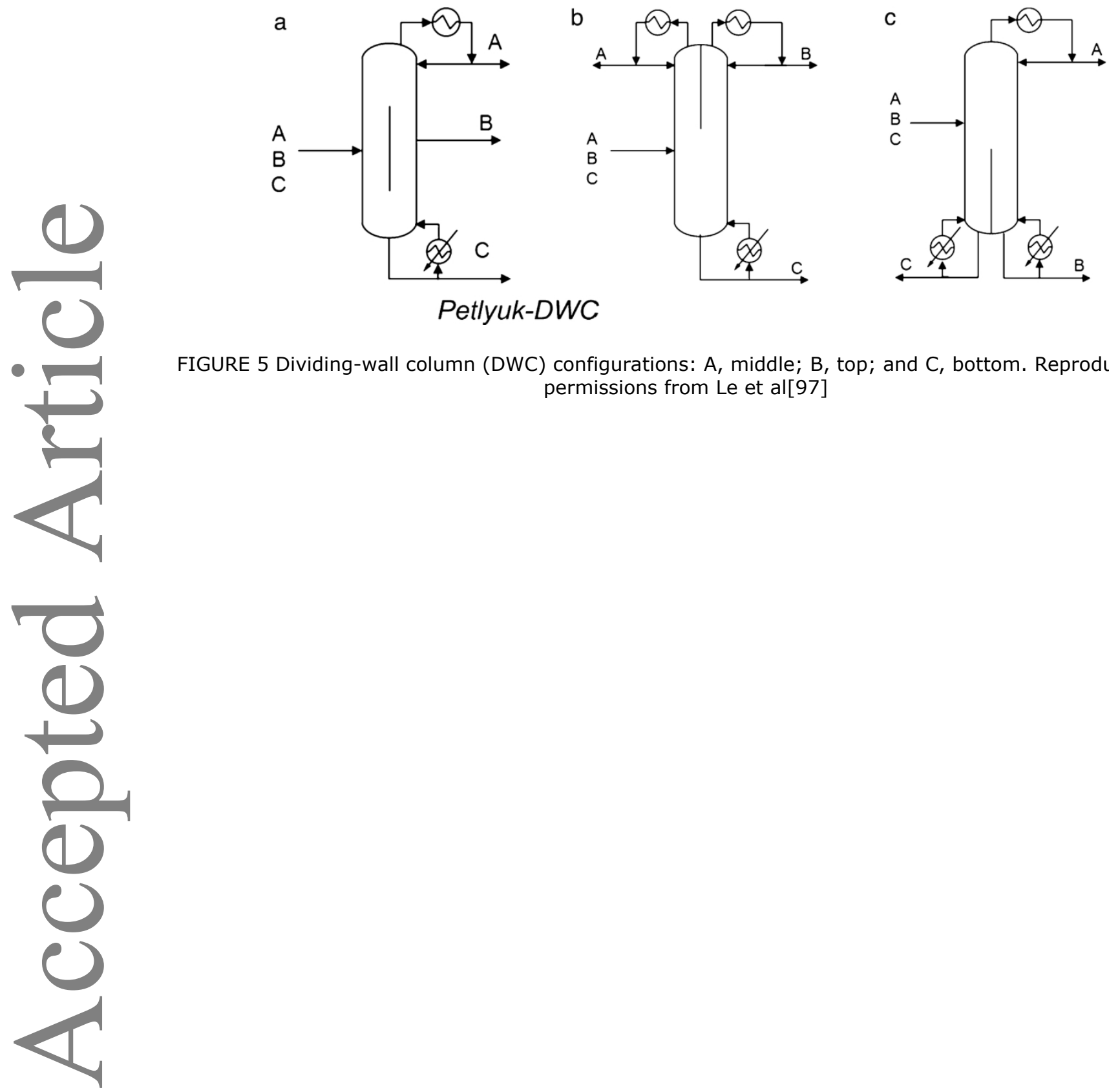

FIGURE 5 Dividing-wall column (DWC) configurations: A, middle; B, top; and C, bottom. Reproduced with permissions from Le et al[97] 


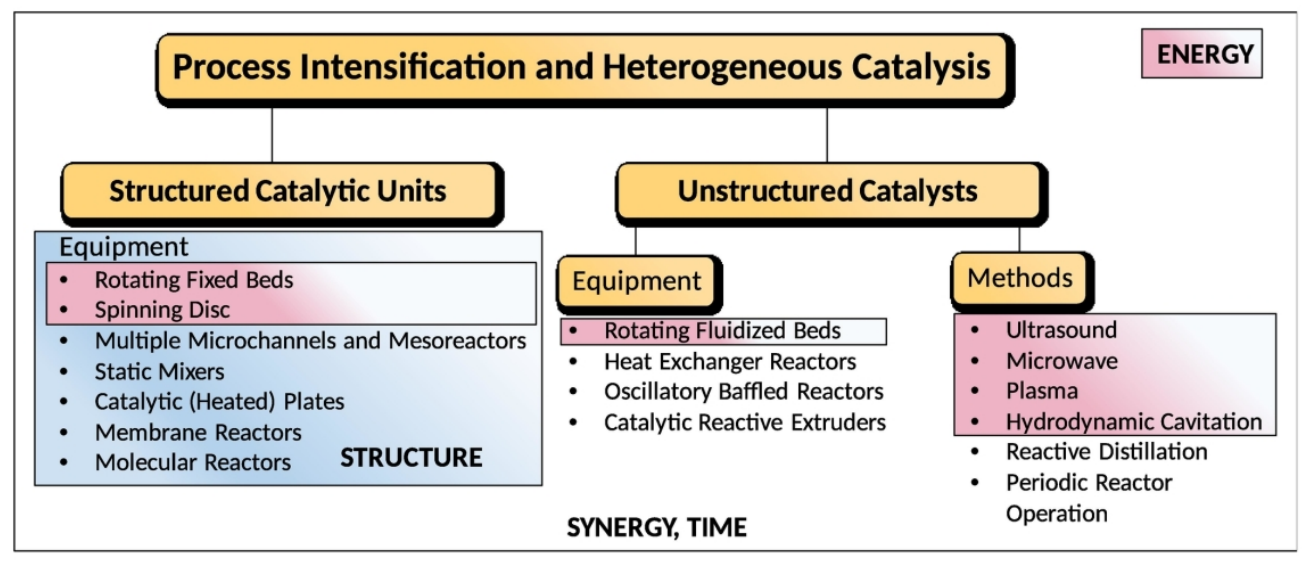

FIGURE 6 The relationship between process intensification and heterogeneous catalysis. Reproduced with permissions from Boffito and Van Gerven[48] 


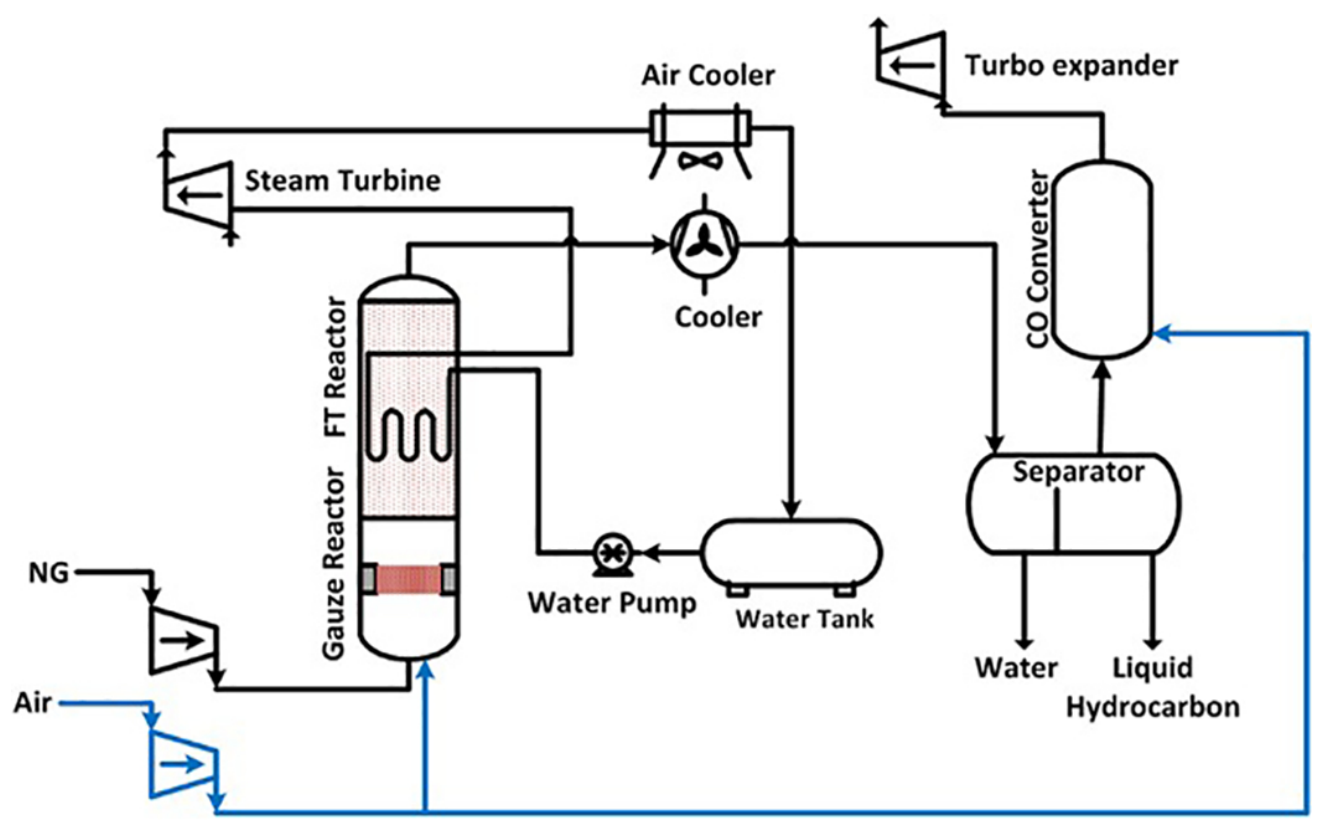

FIGURE 7 Process flow diagram of a gas-to-liquid plant with tandem methane catalytic partial oxidation (CPOX) and Fischer-Tropsch for clean fuels production. Reproduced with permissions from Trevisanut et al[105] 


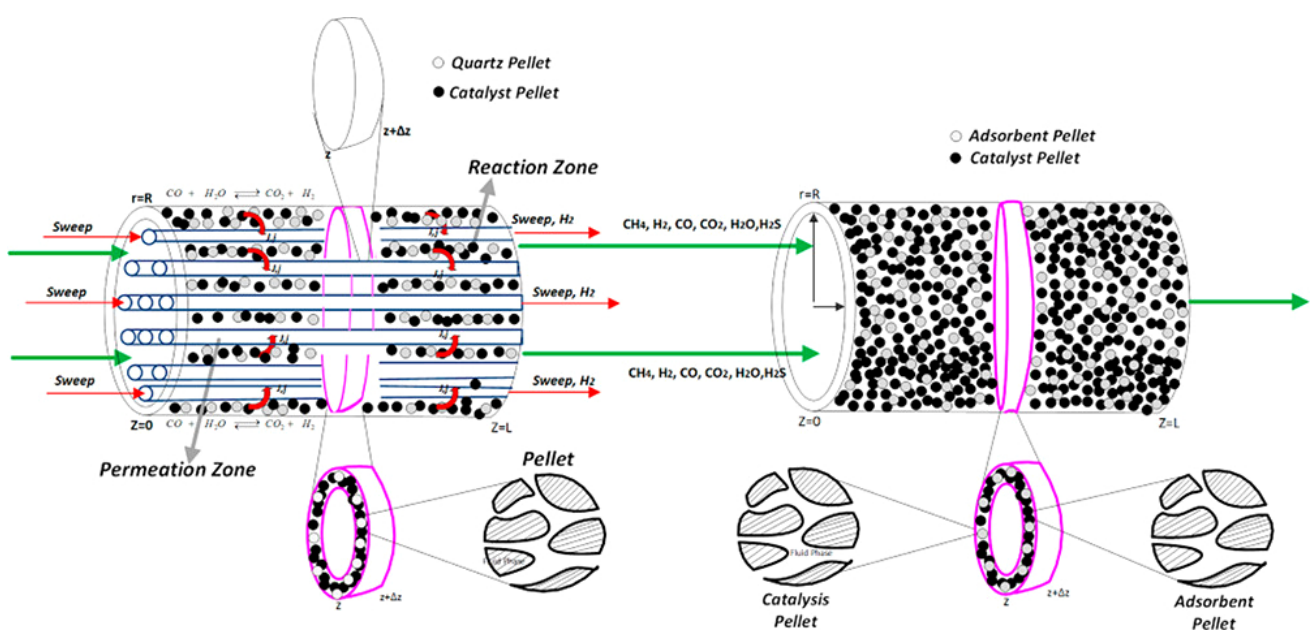

FIGURE 9 A membrane reactor in tandem with and adsorptive reactor (MR-AR) in integrated gasification and combined cycle (IGCC) power plants produce more power and have lower capital cost compared traditional pre-combustion carbon capture systems. Published with permissions from Pichardo et al[114] 

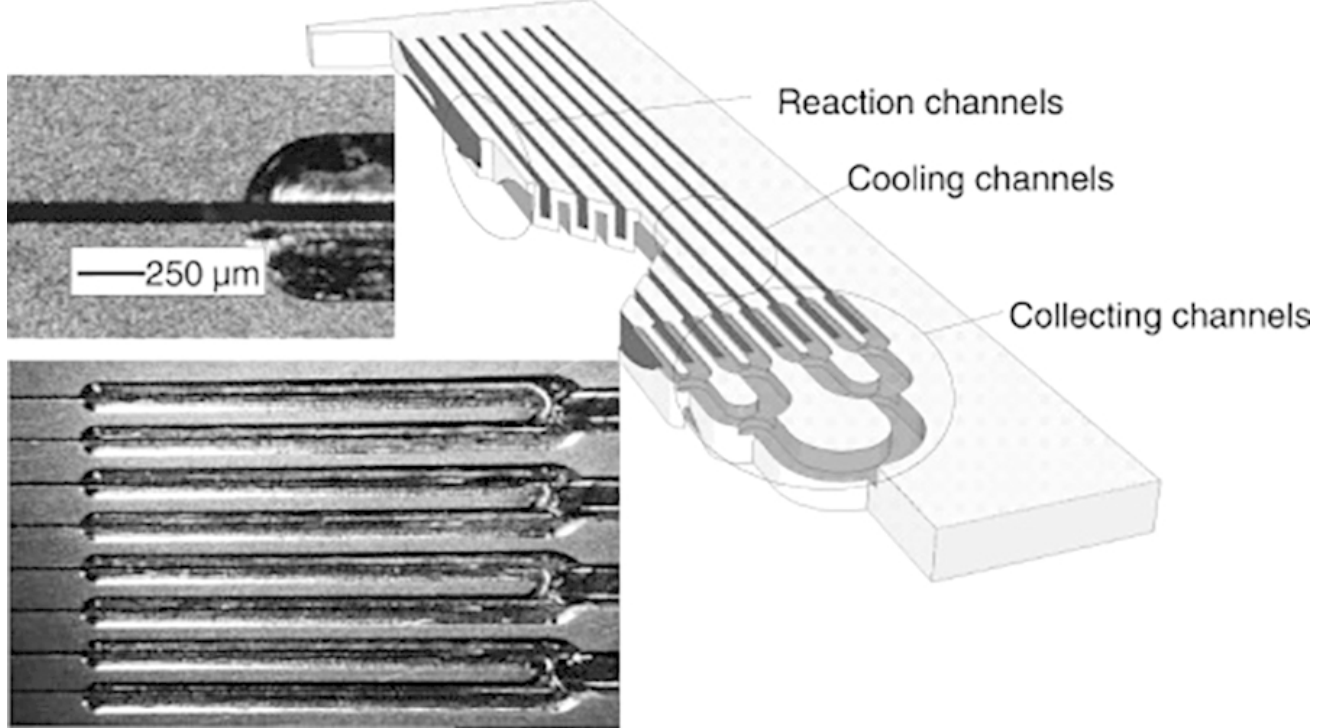

FIGURE 10 Microstructured reactor with mixer, residence-time module, and heat exchanger (IMM for BASF for the highly exothermic synthesis of a vitamin precursor).[122, 123]. Reproduced with permissions from Jähnisch et al[122] 


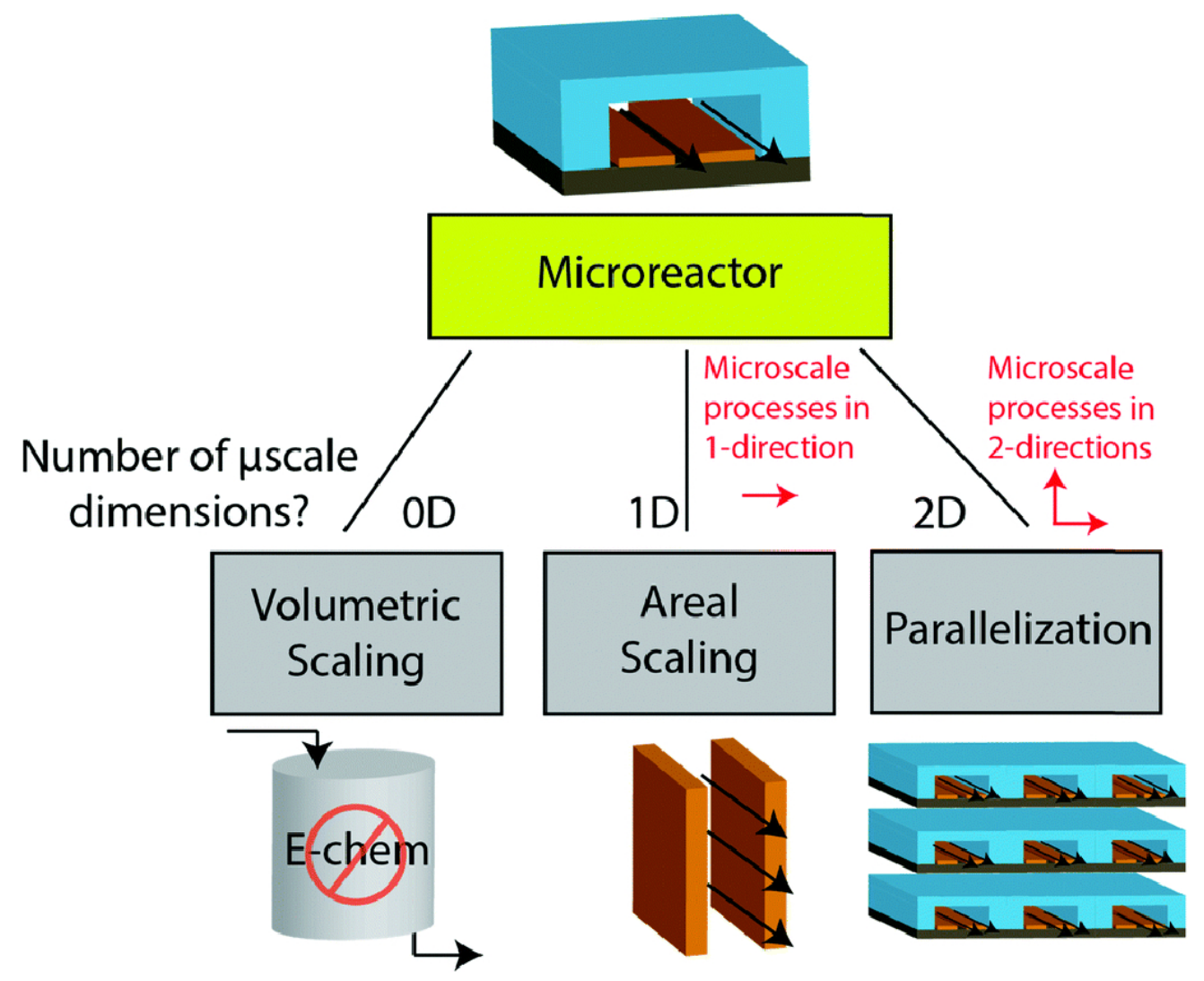

FIGURE 11 Scaling strategies for increasing the throughput of microfluidic reactors. Volumetric scaling is often used for homogeneous chemical processes where the dimensionality of the reactor does not affect the reactions (OD). Areal scaling can be applied if all the limiting transport processes that require microscale path lengths can be carried out in one dimension (1D). A parallelization strategy of microdevices is appropriate when the system involves more than one dimension in the microscale $(\geq 2 \mathrm{D})$. E-chem $=$ electrochemical reaction. However, the authors also state that for micro-reactors numbering-up is challenging and other options should be sought if possible, in particular for multiphase systems.[121] Published by The Royal Society of Chemistry. 

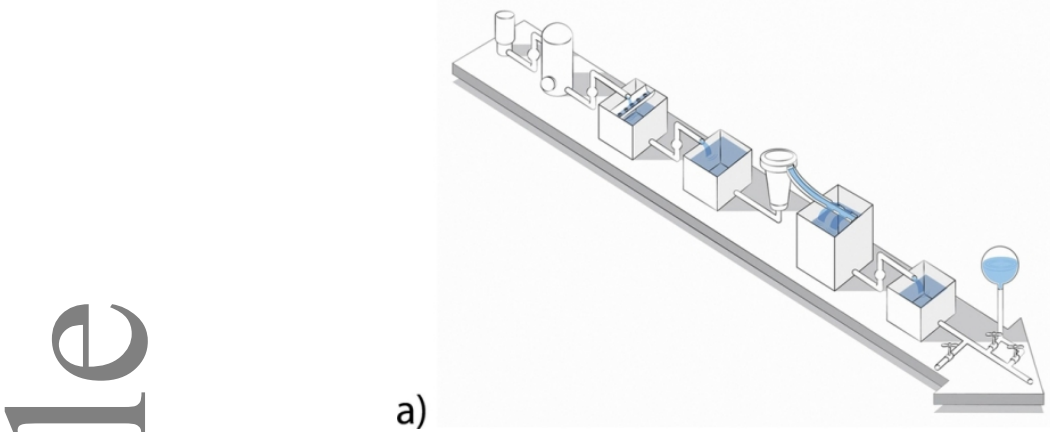

b)

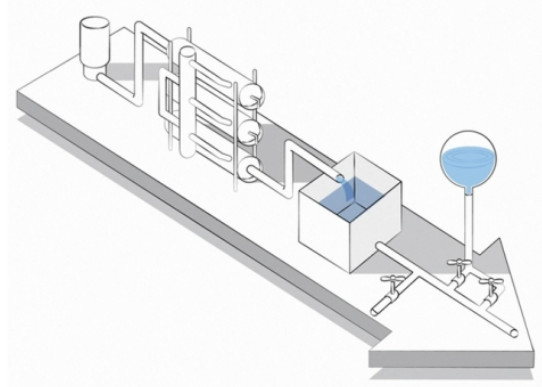

FIGURE 12 A, Traditional water treatment train with seven steps (filtration, aeration, sand filtration, softening, second aeration, second sand filter step, active carbon filter, and a final ultraviolet disinfection step; and B, novel process intensification called One-Step Reverse Osmosis (OSRO) with only four steps. Reproduced with permissions from Fernandez-Rivas et al[78] 